Eletrodos de Carbono Cerâmico Modificados com Óxido Misto de Estanho e Nióbio com Potencial Aplicação como Sensores Eletroquímicos

\author{
Ciórcero, J.; Rosa, G. B.; Pessôa, C. A.*
}

Rev. Virtual Quim., 2019, 11 (6), 1853-1871. Data de publicação na Web: 2 de janeiro de 2020

http://rvq.sbq.org.br

\title{
Carbon Ceramic Electrodes Modified With Mixed Oxides of Tin and Niobium for Application as Electrochemical Sensors
}

\begin{abstract}
The insertion of mixed oxides into ceramic carbon electrodes (CCE) from the sol-gel process can provide materials with high purity and homogeneity without phase segregation and high stability, combining both mechanical and chemical properties of silica gel with the chemical properties of metal oxide (MxOy). This feature can provide a device with interesting properties, since the homogeneous distribution of the oxides, can minimize the resistivity provided by the silicaóxi network. In addition, the presence of acidic sites allows the immobilization of different electron mediators, which remain stable on the surface of the CCE, increasing the selectivity and sensitivity of such electrodes. Thus, the objective of this work was to incorporate the niobium oxide (V) ( $\mathrm{Nb}_{2} \mathrm{O}_{5}$ ) into the structure of carbon ceramic electrode modified with tin oxide, $\mathrm{CCE} / \mathrm{SiO}_{2}-\mathrm{SnO}_{2}$, in order to reduce even more the internal electrical resistance of the silica matrix, obtaining the carbon ceramic electrode, designated as $\mathrm{CCE} / \mathrm{SiO}_{2}-\mathrm{SnO} 2-\mathrm{Nb}_{2} \mathrm{O}_{5}$. The obtained material was characterized by Fourier transform infrared spectroscopy (FTIR), which indicated that the $\mathrm{SiO} 2$ network is little disturbed by the incorporation of the oxides, showing evidence of Si-O-M bond formation (where $\mathrm{M}=\mathrm{Sn}$ or $\mathrm{Nb}$ ), which was corroborated by the $\mathrm{X}$ ray photoelectron spectroscopy technique (XPS). Raman spectra have shown an increase in the intensity ratio between the $D$ and $G$ bands (ID/IG), indicating that as the oxides are inserted into the SiO2 structure, an increase in the degree of system disorder occurs. From the morphological techniques, SEM (Scanning electron microscopy) and EDS (Energy dispersive $\mathrm{X}$ ray spectroscopy), it was shown that $\mathrm{Sn}$ and $\mathrm{Nb}$ were well distributed over the surface. $\mathrm{ECC} / \mathrm{SiO} 2-\mathrm{SnO}_{2}$ and $\mathrm{ECC} / \mathrm{SiO}_{2}-\mathrm{SnO} \mathrm{O}_{2}-\mathrm{Nb}_{2} \mathrm{O}_{5} \mathrm{presented}$ better voltammetric responses than the systems without these oxides $\left(\mathrm{ECC} / \mathrm{SiO}_{2}\right)$, showing a diffusional mass transfer process with stable current responses after 100 voltammetric cycles, with variation of Ipa and Ipc below $5 \%$. These results demonstrate the potential use of these materials in the development of sensors.
\end{abstract}

Keywords: Ceramic carbon electrodes; mixed oxides; tin oxide; niobium oxide; electrochemical sensors.

\section{Resumo}

A inserção de óxidos mistos em eletrodos de carbono cerâmico (ECC) a partir do processo sol-gel pode proporcionar materiais com alta pureza e homogeneidade, sem a segregação de fases e alta estabilidade, combinando as propriedades mecânicas e químicas da sílica gel com as propriedades químicas dos óxidos metálicos ( $\mathrm{MxOy}$ ). Esse recurso pode fornecer ao dispositivo propriedades interessantes, uma vez que a distribuição homogênea dos óxidos, pode minimizar a resistividade proporcionada pela rede de sílica. Além disso, a inserção de sítios ácidos possibilita a imobilização de diferentes mediadores de elétrons, os quais permanecem estáveis sobre a superfície dos ECC aumentando a seletividade e sensibilidade de tais eletrodos. Desta forma, neste trabalho teve-se como objetivo incorporar o óxido de nióbio (V) (Nb2O5) no ECC/SiO2-SnO2, de modo reduzir ainda mais a resistência elétrica interna da matriz de sílica, obtendo-se o eletrodo de carbono cerâmico, designado como ECC/SiO2-SnO2-Nb2O5. O material obtido foi caracterizado por Espectroscopia na região do infravermelho (FTIR), a qual indicou que a rede de SiO2 é pouco perturbada pela incorporação dos óxidos, mostrando evidências da formação da ligação Si-O-M (onde $\mathrm{M}=\mathrm{Sn}$ ou $\mathrm{Nb}$ ), a qual foi corroborada pela técnica de Espectroscopia de fotoelétrons excitados por raios X (XPS). Espectros Raman evidenciaram um aumento da relação de intensidade entre as bandas $D$ e $G$ (ID/IG), indicando que a medida que os óxidos são inseridos na estrutura da SiO2, ocorre um aumento do grau de desordem do sistema. A partir das técnicas morfológicas, MEV (Microscopia eletrônica de varredura) e EDS (Espectroscopia de energia dispersiva de raios X) evidenciou-se que o $\mathrm{Sn}$ e o Nb mostraram-se bem distribuídos pela superfície. O ECC/SiO2-SnO2 e o ECC/SiO2-SnO2-Nb2O5 apresentaram melhores respostas voltamétricas que os sistemas sem a adição destes (ECC/SiO2), apresentando um processo difusional de transferência de massa e com respostas de corrente estáveis após 100 ciclos voltamétricos, com variação de Ipa e Ipc abaixo de $5 \%$. Tais resultados demonstram a potencialidade de utilização destes materiais no desenvolvimento de sensores.

Palavras-chave: Eletrodos de carbono cerâmico; óxidos mistos; óxido de estanho; óxido de nióbio; sensores eletroquímicos.

\footnotetext{
* Universidade Estadual de Ponta Grossa, Departamento de Química, Campus Uvaranas, Praça Santos Andrade 01, CEP 84030-000, Ponta Grossa-PR, Brasil.

M christiana.pessoa@pq.cnpq.br

DOI: $10.21577 / 1984-6835.20190129$
} 


\section{Eletrodos de Carbono Cerâmico Modificados com Óxido Misto de Estanho e Nióbio com Potencial Aplicação como Sensores Eletroquímicos Juliane Ciórcero, ${ }^{a}$ Guilherme Bonifácio Rosa, ${ }^{a}$ Christiana A. Pessôa*}

Universidade Estadual de Ponta Grossa, Departamento de Química, Campus Uvaranas, Praça Santos Andrade 01, Centro, CEP 84030-000, Ponta Grossa-PR, Brasil.

christiana.pessoa@pq.cnpq.br

\section{Introdução}

\section{Parte Experimental}

2.1. Reagentes e soluções

2.2. Síntese do compósito $\mathrm{ECC} / \mathrm{SiO}_{2}-\mathrm{Nb}_{2} \mathrm{O}_{5}$

2.3. Síntese do compósito $\mathrm{ECC} / \mathrm{SiO}_{2}-\mathrm{SnO}_{2}$

2.4. Síntese do compósito $\mathrm{ECC} / \mathrm{SiO}_{2}-\mathrm{SnO}_{2}-\mathrm{Nb}_{2} \mathrm{O}_{5}$

2.5. Espectroscopia na Região do Infravermelho (FTIR)

2.6. Espectroscopia RAMAN

2.7. Espectroscopia de fotoelétrons de Raios $X$

2.8. Difração de Raios X (DRX)

2.9. Caracterização morfológica por MEV/FEG

2.10. Estudos voltamétricos

\section{Resultados e Discussão}

\subsection{Espectroscopia FTIR}

3.2. Espectroscopia RAMAN

3.3. Espectroscopia totoeletrônica de Raios X (XPS)

3.4. Difração de Raios X (DRX)

3.5. Caracterização morfológica por MEV/FEG

3.6. Estudos voltamétricos dos compósitos $\mathrm{ECC} / \mathrm{SiO}_{2}-\mathrm{SnO}_{2}, \mathrm{ECC} / \mathrm{SiO}_{2}-\mathrm{Nb}_{2} \mathrm{O}_{5}$ e ECC/SiO $\mathrm{Sin}_{2}-\mathrm{SnO}_{2}-\mathrm{Nb}_{2} \mathrm{O}_{5}$

\section{Conclusão}

\section{Introdução}

Os eletrodos de carbono cerâmico (ECC) foram propostos pela primeira vez por Lev et al. em 1994, os quais tem apresentado um grande desenvolvimento desde a última década. ${ }^{1}$ Estes têm atraído considerável interesse por pesquisadores da área da Eletroanalítica, pois constituem materiais porosos que permitem um aumento na superfície ativa, além de possibilitar a obtenção de eletrodos com alta

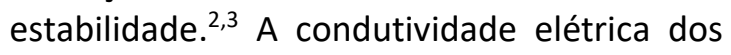
mesmos é obtida a partir da adição de um material de carbono (o qual pode ser grafite, 
nanotubos de carbono, carbono vítreo, grafeno, dentre outros). Como o material carbonáceo permanece entrapeado na matriz sol-gel de sílica, estes exibem uma alta estabilidade mecânica. Além disso, as propriedades químicas da sílica podem ser manipuladas a partir da utilização de diferentes monômeros. Dentre outras características, estes ainda podem ser facilmente modificados com complexos inorgânicos, ${ }^{4}$ óxidos metálicos, ${ }^{5,7}$ compostos $\operatorname{orgânicos}^{8,9}$ e enzimas, ${ }^{10-12}$ proporcionando um incremento nas propriedades eletrocatalíticas de tais eletrodos. Os ECCS podem ser modificados a partir da adição de uma solução de um modificador na superfície do eletrodo ${ }^{13}$ ou pela eletrodeposição de nanopartículas (prata, ouro, platina) na superfície dos mesmos. ${ }^{14,15}$ Geralmente, as modificações na superfície permitem obter sensores com alta sensibilidade, no entanto estes apresentam a desvantagem da baixa estabilidade das espécies eletroativas as quais tendem a lixiviar para eletrólito suporte durante as medidas eletroquímicas ou ainda; se a sua superfície for danificada, a preparação da nova camada modificadora é necessária, pois resultados não reprodutíveis podem ser obtidos. Tal problema pode ser solucionado a partir da modificação do bulk do ECC, permitindo a renovação da superfície a partir de um simples procedimento de polimento.

A inserção de óxidos mistos em ECC através do processo sol-gel tornou-se um processo efetivo, pois proporciona a formação de materiais com alta pureza e homogeneidade, sem a segregação de fases. Esse recurso é muito importante pois permite a distribuição homogênea dos óxidos em todo bulk do ECC, - que pode proporcionar propriedades interessantes ao material. Por exemplo, a resistividade devido à presença de sílica (característica indesejada) pode ser minimizada com a inserção de óxidos metálicos. ${ }^{16}$ Adicionalmente, a presença de sítios ácidos possibilita a imobilização de diferentes mediadores de elétrons, os quais permanecem estáveis sobre a superfície dos $E C C$, além de aumentar a seletividade e sensibilidade de tais dispositivos. Várias configurações de ECCs têm sido obtidas com diferentes óxidos metálicos, tais como $\mathrm{SiO}_{2}$ $\mathrm{SnO}_{2},{ }^{17} \mathrm{SiO}_{2}-\mathrm{ZrO}_{2},{ }^{18} \mathrm{SiO}_{2}-\mathrm{Nb}_{2} \mathrm{O}_{5},{ }^{19},{ }^{20} \mathrm{SiO}_{2}-\mathrm{TiO}_{2}{ }^{21}$ e $\mathrm{SiO}_{2}-\mathrm{SnO}_{2}-\mathrm{Sb}_{2} \mathrm{O}_{5}{ }^{22}$

Dentre os óxidos incorporados na estrutura dos ECCs, o óxido de estanho (II) $\left(\mathrm{SnO}_{2}\right)$ é um dos mais utilizados. $\mathrm{O} \mathrm{SnO}_{2}$, conhecido como cassiterita, é a forma mais comum em que se encontra o estanho ( $\mathrm{Sn}$ ) na natureza. Este material é um semicondutor natural do tipo-n, com um alto valor de band gap de energia, com aproximadamente $3,6 \mathrm{eV}$. O óxido misto formado com $\mathrm{SiO}_{2} / \mathrm{SnO}_{2}$ apresenta características importantes, como alta estabilidade térmica e química, presença de sítios ácidos, alta porosidade e diminuição da resistência interna da matriz. ${ }^{23,24}$ Canevari et al. imobilizaram a ftalocianina de cobalto (CoPc) na superfície da matriz de um eletrodo de óxido misto obtido pelo método sol-gel $\left(\mathrm{SiO}_{2} / \mathrm{SnO}_{2}\right)$. A adição de $\mathrm{SnO}_{2}$ reduziu consideravelmente a resistência elétrica da matriz, apresentando assim potencial para a preparação de um sensor eletroquímico para o ácido oxálico. ${ }^{25} \mathrm{Em}$ outro trabalho, o ECC modificado com óxido de estanho foi obtido utilizando o processo sol-gel a partir da adição de um precursor inorgânico, $\mathrm{SnCl}_{4} .5 \mathrm{H}_{2} \mathrm{O}$, e $\mathrm{HCl}$ como catalisador. Um polímero híbrido orgânico-inorgânico do tipo silsesquioxano, solúvel em meio aquoso, foi imobilizado na superfície do ECC, e suas características morfológicas e estruturais foram estudadas no desenvolvimento do sensor eletroquímico para nitrito. ${ }^{24}$

É conhecido da literatura que a adição de $\mathrm{Nb}_{2} \mathrm{O}_{5}$ em cerâmicas a base de $\mathrm{SnO}_{2}$ proporciona um aumento da condutividade elétrica das mesmas. ${ }^{16}$ Tal aumento na condutividade é atribuído ao fato do íon pentavalente atuar como um doador de elétrons no retículo do $\mathrm{SnO}_{2}$, uma vez vacâncias de $\mathrm{Sn}$ são criadas quando o $\mathrm{Nb}^{5+}$ substitui os íons $\mathrm{Sn}^{4+} \cdot{ }^{16} \mathrm{O} \quad \mathrm{Nb}_{2} \mathrm{O}_{5}$ é um semicondutor tipo " $\mathrm{n}$ " com band gap variando de 3,1 a 4,0 eV. Este possui sítios ácidos de Brönsted e Lewis, com alta acidez $\left(H_{0}=-5,6\right)$ e características anfotéricas, podendo ser dissolvido tanto em ácidos fortes como em bases fortes. A ampla variação da ligação $\mathrm{Nb}-\mathrm{O}$, de estruturas, além da possibilidade de combinação com outros óxidos resultam em propriedades únicas, as quais possibilitam a utilização destes óxidos como 
catalisadores de diversas reações químicas. ${ }^{26}$ Desta forma, como a incorporação do $\mathrm{Nb}_{2} \mathrm{O}_{5}$ no $\mathrm{ECC} / \mathrm{SiO}_{2}-\mathrm{SnO}_{2}$ pode proporcionar uma redução ainda maior na resistência elétrica interna da matriz de sílica, o objetivo deste trabalho consistiu em obter um eletrodo de carbono cerâmico modificado com óxido misto de $\mathrm{SnO}_{2}$ e $\mathrm{Nb}_{2} \mathrm{O}_{5}$, designado como $\mathrm{ECC} / \mathrm{SiO}_{2}-\mathrm{SnO}_{2}-\mathrm{Nb}_{2} \mathrm{O}_{5}$ a partir do $\mathrm{ECC} / \mathrm{SiO}_{2}-\mathrm{SnO}_{2}$, cuja síntese foi anteriormente otimizada. ${ }^{5} \mathrm{O}$ material obtido foi caracterizado por técnicas espectroscópicas e morfológicas e as propriedades eletroquímicas do mesmo comparadas com os eletrodos de carbono cerâmicos, $\mathrm{ECC} / \mathrm{SiO}_{2}, \mathrm{ECC} / \mathrm{Nb}_{2} \mathrm{O}_{5}$ e $\mathrm{ECC} / \mathrm{SiO}_{2}-\mathrm{SnO}_{2}$, com o objetivo de aplicá-los no desenvolvimento de sensores eletroquímicos.

\section{Parte Experimental}

\subsection{Reagentes e soluções}

Metiltrimetoxissilano (MTMOS), diacetato de dibutilestanho $\left.\left(\left(\mathrm{C}_{4} \mathrm{H}_{11}\right)_{2}\right) \mathrm{Sn}\left(\mathrm{OCOCH}_{3}\right)_{2}\right)$, $\mathrm{NbCl}_{5}$ e pó de grafite foram adquiridos da Sigma-Aldrich. O metanol 99,8 \% foi obtido a partir de Neon e ácido clorídrico $38 \%$ da Dinâmica. Todos os demais produtos químicos utilizados neste trabalho são de grau analítico e utilizados sem purificação adicional.

\subsection{Síntese dos materiais compósitos}

Para preparação do compósito $\mathrm{ECC} / \mathrm{SiO}_{2}-\mathrm{Nb}_{2} \mathrm{O}_{5}$ utilizou-se $\mathrm{NbCl}_{5}$ como precursor inorgânico. Este foi preparado a partir da adição de $20 \mathrm{~mL}$ de MTMOS, o qual foi pré-hidrolisado em uma solução de $20 \mathrm{~mL}$ de metanol, 0,24 mL de HCl $38 \%$ e $1,84 \mathrm{~mL}$ de $\mathrm{H}_{2} \mathrm{O}$, durante o período de 3 horas. À solução resultante adicionou-se $1,6 \mathrm{~g}$ de $\mathrm{NbCl}_{5}$, em atmosfera inerte de nitrogênio sob refluxo e agitação por 45 minutos. Em seguida, aqueceu-se o sistema a $60{ }^{\circ} \mathrm{C}$ por mais 45 minutos. Após adicionou-se $22 \mathrm{~g}$ de pó de grafite e $20 \mathrm{~mL}$ de metanol e manteve-se o sistema a $60{ }^{\circ} \mathrm{C}$ por mais 1 hora. Após este período elevou-se a temperatura para $80{ }^{\circ} \mathrm{C}$ para total evaporação do solvente. $\mathrm{O}$ xerogel formado foi colocado em seringa plástica e adicionado contato elétrico de cobre para a realização das medidas eletroquímicas. Estes foram levados para dessecador por 60 dias. Para a síntese do compósito $\mathrm{ECC} / \mathrm{SiO}_{2}-\mathrm{SnO}_{2}$, este foi preparado de acordo com procedimento já descrito na literatura, ${ }^{5}$ similar ao descrito para o $\mathrm{ECC} / \mathrm{SiO}_{2}-\mathrm{Nb}_{2} \mathrm{O}_{5}$, adiconando-se após a reação de hidrólise do MTMOS, 5,4 mL de dibutildiacetato de estanho $\left.\left(\left(\mathrm{C}_{4} \mathrm{H}_{11}\right)_{2}\right) \mathrm{Sn}\left(\mathrm{OCOCH}_{3}\right)_{2}\right)$ dissolvidos em 10 $\mathrm{mL}$ de metanol. Para a obtenção do óxido misto $\left(\mathrm{ECC} / \mathrm{SiO}_{2}-\mathrm{SnO}_{2}-\mathrm{Nb}_{2} \mathrm{O}_{5}\right)$, foi adicionado primeiramente $5,4 \mathrm{~mL}$ de dibutildiacetato de estanho e $10 \mathrm{~mL}$ de metanol, mantendo-se sob agitação por 2 horas. Em seguida adicionou-se 1,6 $\mathrm{g}$ de $\mathrm{NbCl}_{5}$ que permaneceu em atmosfera inerte de nitrogênio sob refluxo e agitação por 45 minutos. As demais etapas de preparação do eletrodo compósito foram similares as detalhadamente descritas para o compósito de $\mathrm{ECC} / \mathrm{SiO}_{2}-\mathrm{Nb}_{2} \mathrm{O}_{5}$.

\subsection{Síntese do compósito $\mathrm{ECC} / \mathrm{SiO}_{2}-\mathrm{SnO}_{2}$}

O compósito $\mathrm{ECC} / \mathrm{SiO}_{2}-\mathrm{SnO}_{2}$ foi preparado de acordo com procedimento já descrito na literatura. ${ }^{5}$ Em sistema sob refluxo adicionouse $20 \mathrm{~mL}$ de MTMOS (metiltrimetoxisilano), 20 $\mathrm{mL}$ de metanol e $0,24 \mathrm{~mL}$ de $\mathrm{HCl} 38 \% \mathrm{~m} / \mathrm{m}$ com $1,84 \mathrm{~mL}$ de $\mathrm{H}_{2} \mathrm{O}$. Esta mistura foi mantida inicialmente a temperatura ambiente $\left(25^{\circ} \mathrm{C}\right)$ e então agitada por 3 horas. Posteriormente adicionou-se 5,4 $\mathrm{mL}$ de dibutildiacetato de estanho $\left.\left(\left(\mathrm{C}_{4} \mathrm{H}_{11}\right)_{2}\right) \mathrm{Sn}\left(\mathrm{OCOCH}_{3}\right)_{2}\right)$ e $10 \mathrm{~mL}$ de metanol por 2 horas sob aquecimento a $60{ }^{\circ} \mathrm{C}$. Após este procedimento, adicionou-se $22 \mathrm{~g}$ de pó de grafite mais $10 \mathrm{~mL}$ de metanol e manteve-se nas mesmas condições por mais 2 horas. A temperatura do sistema foi elevada a $80{ }^{\circ} \mathrm{C}$ para evaporação do solvente. Os sistemas obtidos foram colocados em seringa plástica e adicionado contato elétrico de cobre e mantidos em dessecador por 60 dias.

\subsection{Síntese do compósito $\mathrm{ECC} / \mathrm{SiO}_{2}-\mathrm{SnO}_{2}$ -} $\mathrm{Nb}_{2} \mathrm{O}_{5}$

O material compósito $\mathrm{ECC} / \mathrm{SiO}_{2}-\mathrm{SnO}_{2}-$ $\mathrm{Nb}_{2} \mathrm{O}_{5}$ foi preparado a partir da adição de 20 $\mathrm{mL}$ de MTMOS pré-hidrolisado em uma solução de $20 \mathrm{~mL}$ de metanol, $0,24 \mathrm{~mL}$ de $\mathrm{HCl}$ $38 \%$ com $1,84 \mathrm{~mL}$ de $\mathrm{H}_{2} \mathrm{O}$ durante 3 horas. À solução resultante adicionou-se $5,4 \mathrm{~mL}$ de dibutildiacetato de estanho e $10 \mathrm{~mL}$ de 
metanol, mantendo-se sob agitação por 2 horas. Em seguida adicionou-se $1,6 \mathrm{~g}$ de $\mathrm{NbCl}_{5}$ que permaneceu em atmosfera inerte de nitrogênio sob refluxo e agitação por 45 minutos. Aqueceu-se o sistema a $60{ }^{\circ} \mathrm{C}$ por mais 45 minutos e adicionou-se $22 \mathrm{~g}$ de pó de grafite e $20 \mathrm{~mL}$ de metanol e manteve-se em agitação por 1 hora. Em seguida elevou-se a temperatura para $60{ }^{\circ} \mathrm{C}$ para evaporação do solvente. $\mathrm{O}$ xerogel formado foi colocado em seringa plástica e adicionado contato elétrico de cobre para as medidas voltamétricas. Estes foram acondicionados no dessecador para total secagem do compósito.

\subsection{Espectroscopia na Região do Infravermelho (FTIR)}

Os espectros na região do infravermelho (FTIR) dos materiais compósitos foram obtidos utilizando pastilhas de $\mathrm{KBr}$, com $1 \%(\mathrm{~m} / \mathrm{m})$ dos óxidos mistos. Para esta análise utilizou-se o equipamento da Marca, PerkinElmer, Modelo, FT-IR Spectrometer Frontier. As medidas foram realizadas na faixa de $500 \mathrm{~cm}-1$ a $3500 \mathrm{~cm}-1$, com 64 acumulações.

\subsection{Espectroscopia RAMAN}

As análises foram realizadas sob deposição das amostras na forma de pó, sobre uma placa de vidro nas seguintes condições: Laser: $20 \mathrm{~mW}$; Resolução: 9-15 cm $\mathrm{cm}^{-1}$; faixa do espectro de $400 \mathrm{a}$ $4485 \mathrm{~cm}^{-1}$; tempo de integração de 5 s e abertura $-50 \times 1000$.

\subsection{Espectroscopia de Fotoelétrons de Raios X}

As medidas de Espectroscopia de fotoelétrons excitados por raios X (XPS) foram realizadas com o equipamento Thermo $\mathrm{K}$ Alpha (Thermo scientific Inc.). Al Ka (1486 eV) raios $\mathrm{X}$ monocromático com tamanho de abertura (spot size cannon) de $300 \mu \mathrm{m}$. A energia de $200 \mathrm{eV}$ foi utilizada na varredura longa e $50 \mathrm{eV}$ para varredura curta, com resolução de $1 \mathrm{e} 0,01 \mathrm{eV}$, respectivamente. $O$ intervalo de energia foi de 0 a $1300 \mathrm{eV}$. O espectro foi ajustado no programa padrão AVANTAGE 5.89 (Análise química de superfície) para análise de XPS por deconvolução dos picos.

\subsection{Difração de Raios X (DRX)}

Os difratogramas de raios $\mathrm{X}$ foram obtidos pelo difratômetro Rigaku Ultima IV. Para esta análise utilizou-se tubo de $\mathrm{Cu}$, com ângulo de varredura de $5^{\circ}-75^{\circ}$ e velocidade $2^{\circ} / \mathrm{min}$.

\subsection{Caracterização morfológica por MEV/FEG}

A caracterização morfológica dos materiais foi realizada por microscopia eletrônica de varredura (MEV/FEG) com efeito de campo TESCAN modelo MIRA 3. As amostras foram trituradas em almofariz e metalizadas com ouro por "sputtering" com equipamento QUORUM, modelo SC7620.

\subsection{Estudos voltamétricos}

Com o objetivo de caracterizar eletroquimicamente os eletrodos preparados $\left(\mathrm{ECC} / \mathrm{SiO}_{2}, \quad \mathrm{ECC} / \mathrm{SiO}_{2}-\mathrm{Nb}_{2} \mathrm{O}_{5}, \quad \mathrm{ECC} / \mathrm{SiO}_{2}-\mathrm{SnO}_{2}\right.$ e $\mathrm{ECC} / \mathrm{SiO}_{2}-\mathrm{SnO}_{2}-\mathrm{Nb}_{2} \mathrm{O}_{5}$ ) foram obtidos voltamogramas cíclicos na presença de $\left[\mathrm{Fe}(\mathrm{CN})_{6}\right]^{3 / 4}$

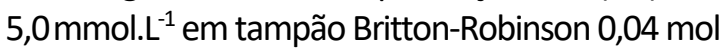
$\mathrm{L}^{-1}$. As medidas eletroquímicas foram realizadas em uma cela eletroquímica de capacidade de $10 \mathrm{~mL}$ utilizando-se como eletrodo de referência $\mathrm{Ag} / \mathrm{AgCl}$. $\mathrm{Ag} \mid \mathrm{AgCl}$ (sat), $\mathrm{KCl}\left(3 \mathrm{~mol} \mathrm{~L}^{-1}\right)$, contra eletrodo de Pt e como eletrodo de trabalho, os ECC. A faixa de potencial foi variada entre $-0,05$ e $0,6 \mathrm{~V}$ a $50 \mathrm{mV} \mathrm{s}^{-1}$. Para estas medidas utilizou-se o Potenciostato/ Galvanostato, AUTOLAB, modelo PGSTAT 100, controlado pelo software GPES 4.9.

\section{Resultados e Discussão}

\subsection{Espectroscopia FTIR}

A técnica de FTIR constitui uma das primeiras estratégias para se verificar a formação de ligações Si-O-M (onde $\mathrm{M}=\mathrm{Sn}$ e $\mathrm{Nb}$ ). A Figura 1a mostra os espectros de FTIR sobrepostos dos materiais compósitos $\mathrm{ECC} / \mathrm{SiO}_{2}-\mathrm{Nb}_{2} \mathrm{O}_{5}, \mathrm{ECC} / \mathrm{SiO}_{2}-$ $\mathrm{SnO}_{2}$ e ECC/SiO ${ }_{2}-\mathrm{SnO}_{2}-\mathrm{Nb}_{2} \mathrm{O}_{5}$. Todos os espectros apresentaram as principais bandas características referentes ao esqueleto de sílica, vSi-O-Si $\mathrm{i}_{\text {ayym }} \mathrm{e}$ vSi-O-Sisym, não ocorrendo deslocamentos significativos entre as mesmas para os diferentes 
materiais. Tais resultados indicam que a estrutura da rede de $\mathrm{SiO}_{2}$ é apenas levemente perturbada pela presença dos óxidos metálicos e que estes encontram-se dispersos na matriz. ${ }^{27}$ As principais atribuições das bandas dos espectros vibracionais para os diferentes materiais de carbono cerâmico estão descritas na Tabela 1.
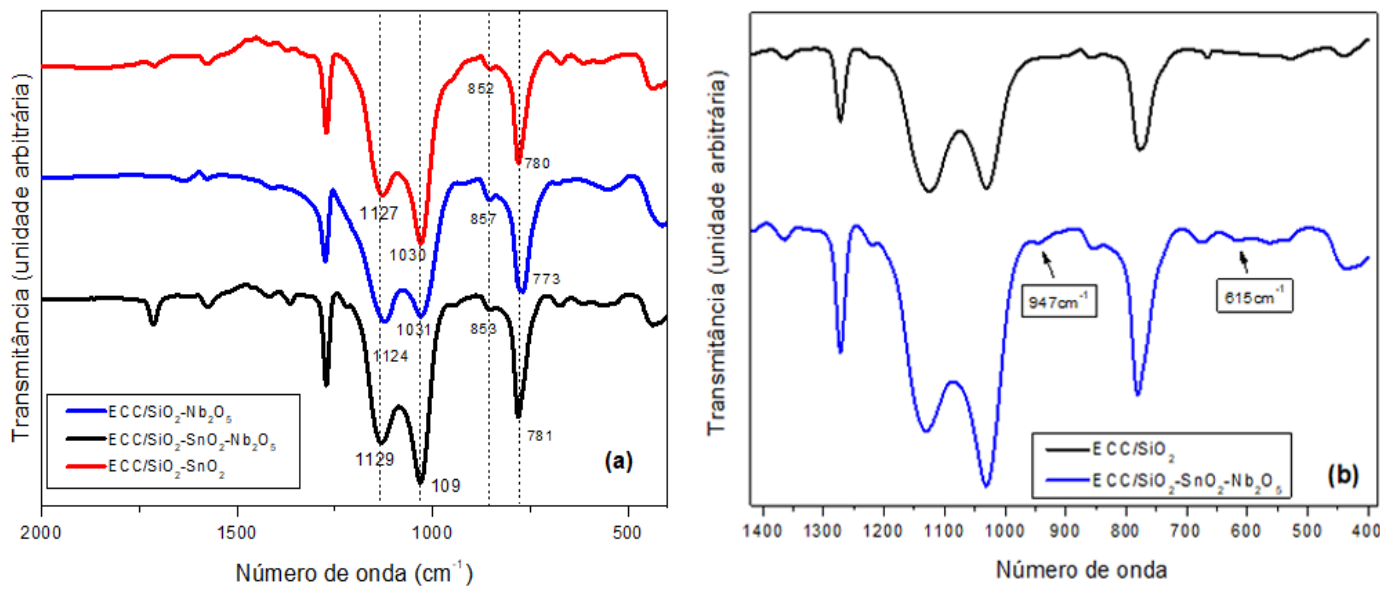

Figura 1. (A) Espectros de FTIR para os sistemas $\mathrm{ECC} / \mathrm{SiO}_{2}-\mathrm{Nb}_{2} \mathrm{O}_{5}, \mathrm{ECC} / \mathrm{SiO}_{2}-\mathrm{SnO}_{2}$ e ECC/SiO $2^{-}$ $\mathrm{SnO}_{2}-\mathrm{Nb}_{2} \mathrm{O}_{5}$ e (B) Comparação entre os espectros $\mathrm{ECC} / \mathrm{SiO} \mathrm{O}_{2}$ e $\mathrm{ECC} / \mathrm{SiO}{ }_{2}-\mathrm{SnO}_{2}-\mathrm{Nb}_{2} \mathrm{O}_{5}$

Tabela 1. Atribuições das bandas principais dos espectros vibracionais dos diferentes materiais de carbono cerâmico.

\begin{tabular}{|c|c|c|c|c|c|}
\hline Estiramento & $\begin{array}{c}v M-O-M_{\text {sym }} \\
\text { (onde } M=S n \text {, } \\
N b \text { ) }\end{array}$ & vSi-O-Si asym $_{\text {. }}$ & $\begin{array}{c}\text { vSi-O Ligação } \\
\text { Si-O-M (onde } \\
\text { M=Nb, Sn) }\end{array}$ & $v S i-O-S i_{\text {sym }}$ & $\begin{array}{l}\text { v Si-O do } \\
\text { grupo SiOH }\end{array}$ \\
\hline $\mathrm{ECC} / \mathrm{SiO}_{2}-\mathrm{SnO}_{2}$ & $669 \mathrm{~cm}^{-1}$ & $\begin{array}{l}780 \mathrm{~cm}^{-1} \\
852 \mathrm{~cm}^{-1}\end{array}$ & $620 \mathrm{~cm}^{-1}$ & $\begin{array}{l}1030 \mathrm{~cm}^{-1} \\
1127 \mathrm{~cm}^{-1}\end{array}$ & $954 \mathrm{~cm}^{-1}$ \\
\hline $\begin{array}{l}\mathrm{ECC} / \mathrm{SiO}_{2-} \\
\mathrm{Nb}_{2} \mathrm{O}_{5}\end{array}$ & $681 \mathrm{~cm}^{-1}$ & $\begin{array}{l}768 \mathrm{~cm}^{-1} \\
854 \mathrm{~cm}^{-1}\end{array}$ & $552 \mathrm{~cm}^{-1}$ & $\begin{array}{l}1031 \mathrm{~cm}^{-1} \\
1124 \mathrm{~cm}^{-1}\end{array}$ & - \\
\hline $\begin{array}{l}\mathrm{ECC} / \mathrm{SiO}_{2-} \\
\mathrm{SnO}_{2}-\mathrm{Nb}_{2} \mathrm{O}_{5}\end{array}$ & $672 \mathrm{~cm}^{-1}$ & $\begin{array}{l}781 \mathrm{~cm}^{-1} \\
853 \mathrm{~cm}^{-1}\end{array}$ & $\begin{array}{l}554 \mathrm{~cm}^{-1} \\
615 \mathrm{~cm}^{-1}\end{array}$ & $\begin{array}{l}1029 \mathrm{~cm}^{-1} \\
1129 \mathrm{~cm}^{-1}\end{array}$ & $947 \mathrm{~cm}^{-1}$ \\
\hline
\end{tabular}

Uma forma de verificar a interação entre o alcóxido (MTMOS) e o precursor inorgânico (dibutildiacetato de estanho) é a formação da ligação Sn-O-Si. Esse estiramento apresenta bandas em 620 e $940 \mathrm{~cm}^{-1}$. No espectro de FTIR do $\mathrm{ECC} / \mathrm{SiO}_{2}-\mathrm{SnO}_{2}$, observou-se a banda em 669 $\mathrm{cm}^{-1}$, correspondente ao estiramento simétrico ( $v$ Sn-O-Sn sym $_{\text {}}$ ) e também a banda em $620 \mathrm{~cm}^{-1}$ referente a ligação Sn-O-Si. No espectro do $\mathrm{ECC} / \mathrm{SiO}_{2}-\mathrm{SnO}_{2}-\mathrm{Nb}_{2} \mathrm{O}_{5}$ também podem ser observadas as bandas em $672 \mathrm{~cm}^{-1}$ correspondente ao estiramento simétrico ( $v \mathrm{Sn}$ $\left.\mathrm{O}-\mathrm{Sn}_{\text {sym }}\right)$, a banda em $554 \mathrm{~cm}^{-1}$ referente a $v \mathrm{Si}$ -
$\mathrm{OH}$ no $\mathrm{ECC} / \mathrm{SiO}_{2}-\mathrm{Nb}_{2} \mathrm{O}_{5}$ e em $617 \mathrm{~cm}^{-1}$ referente a ligação $\mathrm{Sn}-\mathrm{O}-\mathrm{Si} .{ }^{28,} 29$

Para uma melhor visualização das bandas referentes aos estiramentos Si-O-M ou M-O$\mathrm{M}$ (onde $\mathrm{M}=\mathrm{Nb}$ ou $\mathrm{Sn}$ ), foram sobrepostos os espectros do apenas do carbono cerâmico (sem incorporação dos óxidos metálicos) designado como $\mathrm{ECC} / \mathrm{SiO}_{2}$ e o $\mathrm{ECC} / \mathrm{SiO}_{2}-\mathrm{SnO}_{2}-$ $\mathrm{Nb}_{2} \mathrm{O}_{5}$ (Figura 1b). A partir da comparação destes pode-se observar uma banda pouco intensa em $615 \mathrm{~cm}^{-1}$, a qual é devido a ligação Si-O-Sn. Verifica-se também a banda em 947 $\mathrm{cm}^{-1}$, referente ao estiramento Si-O do grupo 
$\mathrm{SiOH}$, a qual encontra-se deslocada para frequências mais baixas devido a formação da ligação Si-O-Sn ou Si-O-Nb. ${ }^{30}$

\subsection{Espectroscopia RAMAN}

Na Figura 2, estão mostrados os espectros Raman dos materiais compósitos. A espectroscopia Raman é uma técnica importante para o estudo e caracterização de propriedades estruturais de materiais de carbono bem como verificar a desordem estrutural apresentada pelos mesmos. Os espectros foram realizados em três regiões diferentes do material cerâmico de carbono. Pode ser observado que para todos os espectros houve uma predominância das bandas atribuídas ao carbono grafítico. O $\mathrm{ECC} / \mathrm{SiO}_{2}$ apresentou bandas em 1329 e $1563 \mathrm{~cm}^{-1}$ relacionadas as bandas D e $G$ (sp3 e sp2), respectivamente, dos materiais carbonáceos amorfos. ${ }^{31}$. O sistema $\mathrm{ECC} / \mathrm{SiO}_{2}-\mathrm{SnO}_{2}$ apresentou espectro similar ao $\mathrm{ECC} / \mathrm{SiO}_{2}$, sendo a banda $\mathrm{D}$ observada em $1347 \mathrm{~cm}^{-1}$ e banda $\mathrm{G}$ em $1569 \mathrm{~cm}^{-}$ 1 , a qual apresentou uma intensidade muito maior que a banda $D$, indicando também um baixo grau de desordem. O sistema $\mathrm{ECC} / \mathrm{SiO}_{2}-$ $\mathrm{Nb}_{2} \mathrm{O}_{5}$, apresentou uma banda em $472 \mathrm{~cm}^{-1}$ referente ao silício amorfo, além das bandas $\mathrm{D}$ e G observadas em $1340 \mathrm{~cm}^{-1}$ e $1569 \mathrm{~cm}^{-1}$, respectivamente. Neste sistema mesmo a banda $G$ apresentando maior intensidade que a banda $D$ (associada a desordem estrutural), esta apresentou uma intensidade superior, em torno de 20 vezes maior em relação aos demais compósitos, o que pode ser decorrente da adição do óxido de nióbio na rede da sílica. Este sistema apresentou também a banda em $795 \mathrm{~cm}^{-1}$ devido a ligação $\mathrm{Nb}-\mathrm{O}-\mathrm{Nb}$ da estrutura $\mathrm{NbO}_{4} \cdot{ }^{32} \mathrm{O}$ sistema $\mathrm{SiO}_{2}-\mathrm{Nb}_{2} \mathrm{O}_{5}$ geralmente apresenta espécies isoladas de $\mathrm{NbO}_{4}$, devido à baixa densidade de grupos hidroxilas na superfície do suporte de $\mathrm{SiO}_{2 .}{ }^{33}$

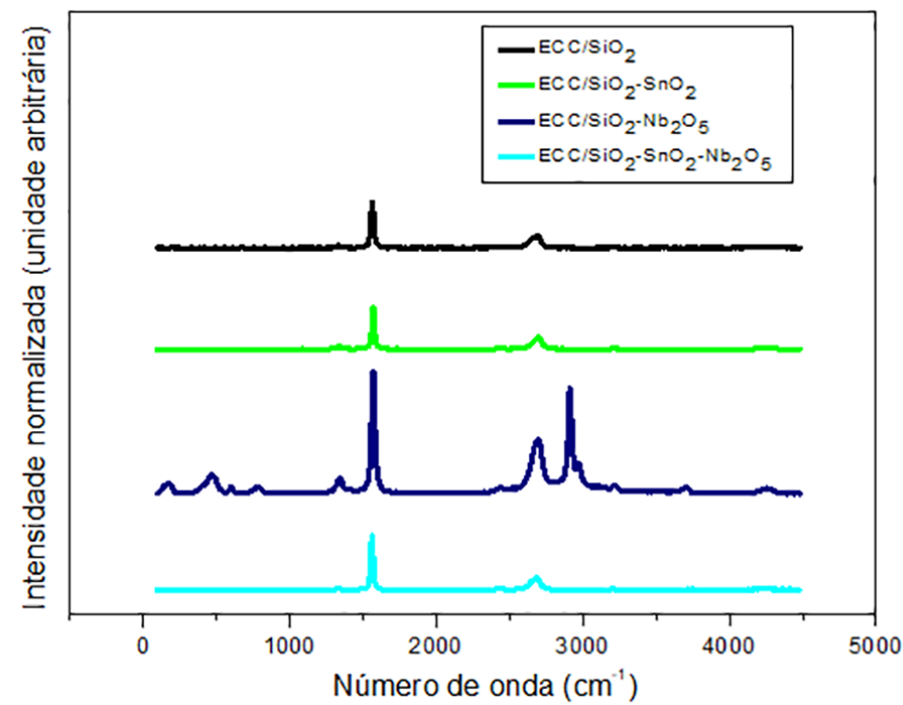

Figura 2. Espectros Raman do sistema $\mathrm{ECC} / \mathrm{SiO}_{2}, \mathrm{ECC} / \mathrm{SiO}_{2}-\mathrm{SnO}_{2}, \mathrm{ECC} / \mathrm{SiO}_{2}-\mathrm{Nb}_{2} \mathrm{O}_{5}$ e $\mathrm{ECC} / \mathrm{SiO}_{2}-$ $\mathrm{SnO}_{2}-\mathrm{Nb}_{2} \mathrm{O}_{5}$, excitado a laser $532 \mathrm{~nm}$

No sistema $\mathrm{ECC} / \mathrm{SiO}_{2}-\mathrm{SnO}_{2}-\mathrm{Nb}_{2} \mathrm{O}_{5}$, a banda $D$ referente ao grafite aparece em $1296 \mathrm{~cm}^{-1}$, e a banda $\mathrm{G}$ em $1443 \mathrm{~cm}^{-1}$, com pequena intensidade quando comparadas as demais. Este sistema apresentou também a banda em $483 \mathrm{~cm}^{-1}$ referente ao silício amorfo e a banda em $793 \mathrm{~cm}^{-1}$ devido a ligação $\mathrm{Nb}-\mathrm{O}-\mathrm{Nb}$ da estrutura $\mathrm{NbO}_{4}{ }^{33}$

Uma relação que podemos utilizar de forma a se estimar o grau de desordem é razão entre as intensidades das bandas $\mathrm{I}_{\mathrm{D}} / \mathrm{I}_{\mathrm{G}}$. Uma razão $I_{D} / I_{G}$ de baixa intensidade indica maior organização e menos defeitos. Para o grafite pirolítico altamente orientado (HOPG) a razão deve ser entre $0,05<\mathrm{I}_{\mathrm{D}} / \mathrm{I}_{\mathrm{G}}<0,3 .{ }^{32}$ Os valores encontrados de $\mathrm{I}_{\mathrm{D}} / \mathrm{I}_{\mathrm{G}}$ foram: $\mathrm{ECC} / \mathrm{SiO}_{2}=$ 0,0452; $\mathrm{ECC} / \mathrm{SiO}_{2}-\mathrm{SnO}_{2}=0,066 ; \quad \mathrm{ECC} / \mathrm{SiO}_{2}-$ $\mathrm{Nb}_{2} \mathrm{O}_{5}=0,1224$ e $\mathrm{ECC} / \mathrm{SiO}_{2}-\mathrm{SnO}_{2}-\mathrm{Nb}_{2} \mathrm{O}_{5}=$ 0,3962 . Pode-se observar um aumento dos valores $\mathrm{I}_{\mathrm{D}} / \mathrm{I}_{\mathrm{G}}$ à medida que os diferentes óxidos 
são incorporados ao compósito, o que é um indicativo de que estes $\left(\mathrm{SnO}_{2}\right.$ e $\left.\mathrm{Nb}_{2} \mathrm{O}_{5}\right)$ proporcionam um aumento do grau de desordem do sistema.

Outros modos Raman de segunda ordem podem ser observados no espectro com frequências acima dos $1700 \mathrm{~cm}-1$, tais como a banda G' (overtone da banda D) localizada no intervalo de $2400-2800 \mathrm{~cm}-1.31$ Neste modo, o espalhamento elástico pelo defeito na banda D é substituído por um espalhamento inelástico por um segundo fônon da mesma frequência, mas com vetor de onda oposto. Esta banda tem sido utilizada para medir os efeitos locais provocados por defeitos ou dopagens nas propriedades eletrônicas e vibracionais de materiais de carbono, pois ela tem se mostrado muito sensível a dopagens substitucionais tipo $n$ ou tipo p mesmo a baixas concentrações $(0,3 \%$ atômico). Pode ser observado que a banda G' apresentou um pequeno deslocamento após a incorporação dos óxidos de estanho e nióbio (em 2702,5 e 2696,0 cm-1, respectivamente) quando comparados aos ECC/SiO2 $(2689,0 \mathrm{~cm}$ 1). No entanto, para o ECC/SiO2-SnO2-Nb2O5, esta banda foi observada em 2687,0 cm-1 não apresentando deslocamento em relação a $\mathrm{ECC} / \mathrm{SiO} 2$.

\subsection{Espectroscopia fotoeletrônica de Raios X (XPS)}

A espectroscopia fotoeletrônica de raios $X$ (XPS) foi empregada para obter algumas informações sobre a composição química e a estrutura da superfície dos sólidos. A Figura 3 apresenta os resultados obtidos por XPS para o $\mathrm{ECC} / \mathrm{SiO} 2-\mathrm{Nb} 2 \mathrm{O} 5$. O pico $\mathrm{C} 1 \mathrm{~s}$ pode ser deconvoluído em três componentes (Figura 3a), o componente correspondente ao carbono da rede planar de grafite aparece em $284,2 \mathrm{eV}$, e os demais em 285,3 eV (C-O); 286,3 (C-O-C); 287,1 $\mathrm{eV}(\mathrm{O}-\mathrm{C}=\mathrm{O}) .32$ Estes apresentaram pequeno deslocamento quando comparado ao pico C1s dos espectros de XPS do $\mathrm{ECC} / \mathrm{SiO}_{2}$ e $\mathrm{ECC} / \mathrm{SiO}_{2}-$ $\mathrm{SnO}_{2 .}{ }^{5}$

Na Figura 3b, está apresentado o espectro de XPS para o nível de 01s, o qual pode ser deconvoluído em um sistema de dois componentes oxigênio, um ligado ao silício ( $\mathrm{Si}$ O), o qual encontra-se em 532, 4 eV e um pico em 530,6 eV, relacionado ao oxigênio ligado ao nióbio (Nb-O). ${ }^{34}$ Comparando-se com o espectro de XPS para o nível de O1s do $\mathrm{Nb}_{2} \mathrm{O}_{5}$ puro, este apresenta um único intenso pico centrado em 529,9 eV, referente aos ânions $\mathrm{O}^{2-}$ vinculados ao nióbio da superfície. ${ }^{35} \mathrm{O}$ deslocamento deste pico para regiões de maior energia para o $\mathrm{ECC} / \mathrm{SiO}_{2}-\mathrm{Nb}_{2} \mathrm{O}_{5}$ é um indicativo da formação da ligação Si-O-Nb.

Na Figura 3c está o espectro XPS na região do Si $2 p$, o qual apresentou um sinal simétrico com valor de energia de ligação em 102,9 eV (Figura 3c), típico de elétrons neste nível. Comparando-se com os sistemas $\mathrm{ECC} / \mathrm{SiO}_{2}$ $(102,9 \mathrm{eV})$ e $\mathrm{ECC} / \mathrm{SiO}_{2}-\mathrm{SnO}_{2}(102,7 \mathrm{eV})$ estes não apresentaram deslocamento significativo (Tabela 2).

Os sinais do dubleto dos elétrons de $\mathrm{Nb} 3 \mathrm{~d}$ para $\mathrm{ECC} / \mathrm{SiO}_{2}-\mathrm{Nb}_{2} \mathrm{O}_{5}$ foram observados em 210,4 eV (Nb3d $\left.\frac{3}{2}\right)$ e 207,6 eV (Nb3d $\frac{5}{2}$ ) (Figura 3d), atribuídos ao $\mathrm{Nb}_{2} \mathrm{O}_{5}$, nióbio com estado de oxidação $\mathrm{Nb}^{+5}{ }^{36}$ Para o óxido de nióbio puro, estes picos encontram-se em 209,8 e 207,1 eV. O pequeno deslocamento observado para os picos do nível $\mathrm{Nb} 3 \mathrm{~d}$ do $\mathrm{ECC} / \mathrm{SiO}_{2}-\mathrm{Nb}_{2} \mathrm{O}_{5}$ em relação ao óxido de nióbio puro pode ser explicado de maneira similar ao constatado para o sistema $\mathrm{ECC} / \mathrm{SiO}_{2}-\mathrm{SnO}_{2}$ em comparação ao $\mathrm{SnO}_{2}$ puro. Como o átomo de silício é mais eletronegativo do que o nióbio, a polarização da ligação Si-O-Nb diminuiu a densidade eletrônica no nióbio, deslocando a energia de ligação do $\mathrm{Nb} 3 \mathrm{~d}$ para valores mais altos. ${ }^{37}$

Para o sistema $\mathrm{ECC} / \mathrm{SiO}_{2}-\mathrm{SnO}_{2}-\mathrm{Nb}_{2} \mathrm{O}_{5}$ (Figuras 4a a $4 \mathrm{e})$, comportamento similar ao $\mathrm{ECC} / \mathrm{SiO}_{2}$ $\mathrm{Nb}_{2} \mathrm{O}_{5}$ foi observado, no entanto com alguns deslocamentos para regiões de maior energia, principalmente em relação ao picos do nível $\mathrm{Nb} 3 \mathrm{~d}$, os quais foram observados em 208,0 eV (Nb3d $\left.\frac{5}{2}\right)$ e 210,8 eV ( $\left.\mathrm{Nb3d}_{2} \frac{3}{2}\right)$. Tais deslocamentos são um indicativo da inserção do óxido de nióbio na rede de sílica modificada com óxido de estanho $\left(\mathrm{SiO}_{2}-\right.$ $\mathrm{SnO}_{2}$ ).

$\mathrm{Na}$ Tabela 2 estão apresentados os resultados de Espectroscopia Fotoeletrônica 
de raios $X$ (XPS) para o $\mathrm{ECC} / \mathrm{SiO}_{2}-\mathrm{Nb}_{2} \mathrm{O}_{5}$ e $\mathrm{ECC} / \mathrm{SiO}_{2}-\mathrm{SnO}_{2}-\mathrm{Nb}_{2} \mathrm{O}_{5}$. Estes foram
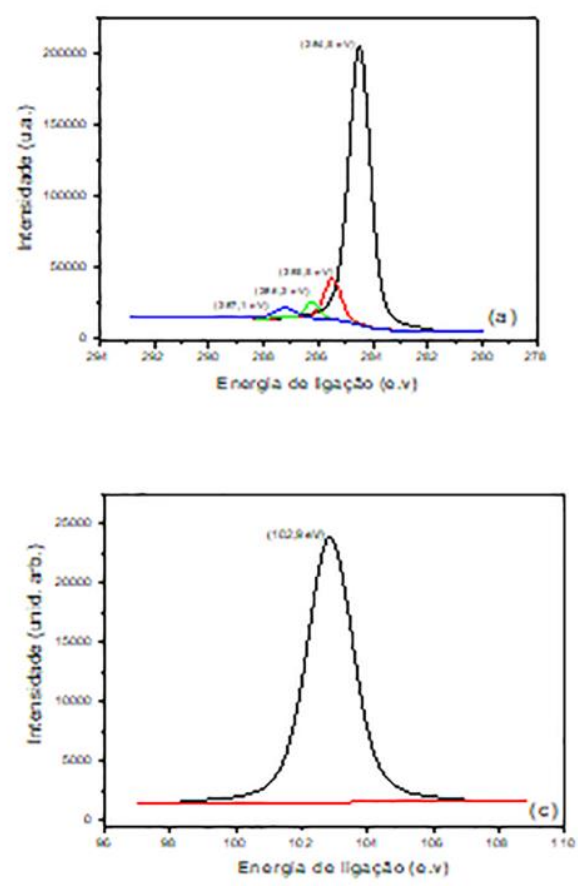

comparados com o XPS do $\mathrm{ECC} / \mathrm{SiO}_{2}-\mathrm{SnO}_{2}$ obtido anteriormente ${ }^{5}$ e do $\mathrm{Nb}_{2} \mathrm{O}_{5}$ puro. ${ }^{35}$
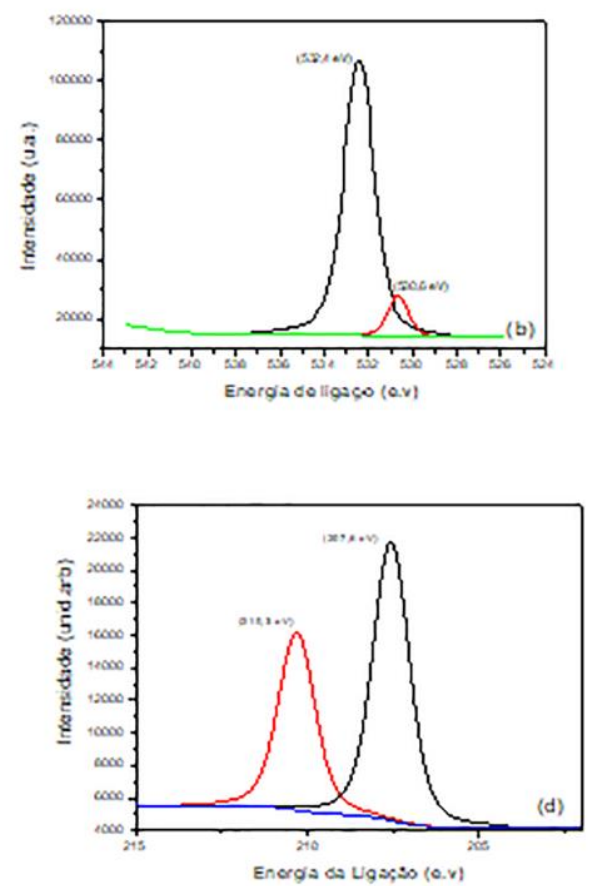

Figura 3. Espectros de XPS do ECC/SiO $-\mathrm{Nb}_{2} \mathrm{O}_{5}$ das regiões (a) C 1s; (b) O 1s; (c) $\mathrm{Si} 2 \mathrm{p}$; e (d) Nb 3d

Tabela 2. Valores de energia de ligação (eV) obtidas a partir dos espectros de XPS para os $\mathrm{ECC} / \mathrm{SiO}_{2}, \mathrm{ECC} / \mathrm{SiO}_{2}-\mathrm{SnO}_{2}-\mathrm{Nb}_{2} \mathrm{O}_{5} \mathrm{ECC} / \mathrm{SiO}_{2}-\mathrm{SnO}_{2}-\mathrm{Nb}_{2} \mathrm{O}_{5} \mathrm{ECC} / \mathrm{SiO}_{2}-\mathrm{SnO}_{2}-\mathrm{Nb}_{2} \mathrm{O}_{5}$

\begin{tabular}{|c|c|c|c|c|c|}
\hline \multirow[b]{2}{*}{ eletrodos } & \multicolumn{5}{|c|}{ Energias de Ligação } \\
\hline & Si2p & $\mathrm{C}$ & O1s & Sn & $\mathrm{Nb}$ \\
\hline & 102,7 & 284,0 & $532,8(\mathrm{Si}-\mathrm{O})$ & 486,6 & - \\
\hline \multirow{3}{*}{$\mathrm{ECC} / \mathrm{SiO}_{2}-\mathrm{SnO}_{2}$} & & 285,1 & 530,3 & $(\mathrm{Sn} 3 \mathrm{~d} 5 / 2)$ & \\
\hline & & 286,3 & $(\mathrm{Sn}-\mathrm{O})$ & 495,0 & \\
\hline & & 289,8 & & $(S n 3 d 3 / 2)$ & \\
\hline \multirow[t]{4}{*}{$\mathrm{Nb}_{2} \mathrm{O}_{5}$} & - & - & 529,9 & - & 207,1 \\
\hline & & & $(\mathrm{Nb}-\mathrm{O})$ & & $\left(\mathrm{Nb} 3 \mathrm{~d}-\frac{5}{2}\right)$ \\
\hline & & & & & 209,8 \\
\hline & & & & & $\left(\mathrm{Nb}_{3} \mathrm{~d}_{2} \frac{3}{2}\right.$ \\
\hline \multirow[t]{6}{*}{$\mathrm{ECC} / \mathrm{SiO}_{2}-\mathrm{Nb}_{2} \mathrm{O}_{5}$} & 102,9 & 284,2 & $532,3(\mathrm{Si}-\mathrm{O})$ & - & 198,6 \\
\hline & & 285,3 & 530.6 & & 200,2 \\
\hline & & 286,3 & $(\mathrm{Nb}-\mathrm{O})$ & & 207,6 \\
\hline & & 287,1 & & & $\left(\mathrm{Nb} 3 \mathrm{~d} \frac{5}{2}\right)$ \\
\hline & & & & & 210,3 \\
\hline & & & & & $\left(\mathrm{Nb}_{3} \mathrm{~d}_{2} \frac{3}{2}\right.$ \\
\hline \multirow[t]{6}{*}{$\mathrm{ECC} / \mathrm{SiO}_{2}-\mathrm{SnO}_{2}-\mathrm{Nb}_{2} \mathrm{O}_{5}$} & 103,0 & 284,5 & $532,6(\mathrm{Si}-\mathrm{O})$ & 486,9 & 199,0 \\
\hline & & 285,5 & 530,8 & $(S n 3 d 5 / 2)$ & 200,7 \\
\hline & & 286,3 & (Nb-O ou & 495,3 & 208,0 \\
\hline & & 287,2 & $\mathrm{Sn}-\mathrm{O})$ & $(\operatorname{Sn} 3 d 3 / 2)$ & $\left(\mathrm{Nb} 3 \mathrm{~d} \frac{5}{2}\right)$ \\
\hline & & & & & 210,8 \\
\hline & & & & & $\left(\mathrm{Nb} 3 \mathrm{~d}^{\frac{3}{2}}\right.$ \\
\hline
\end{tabular}



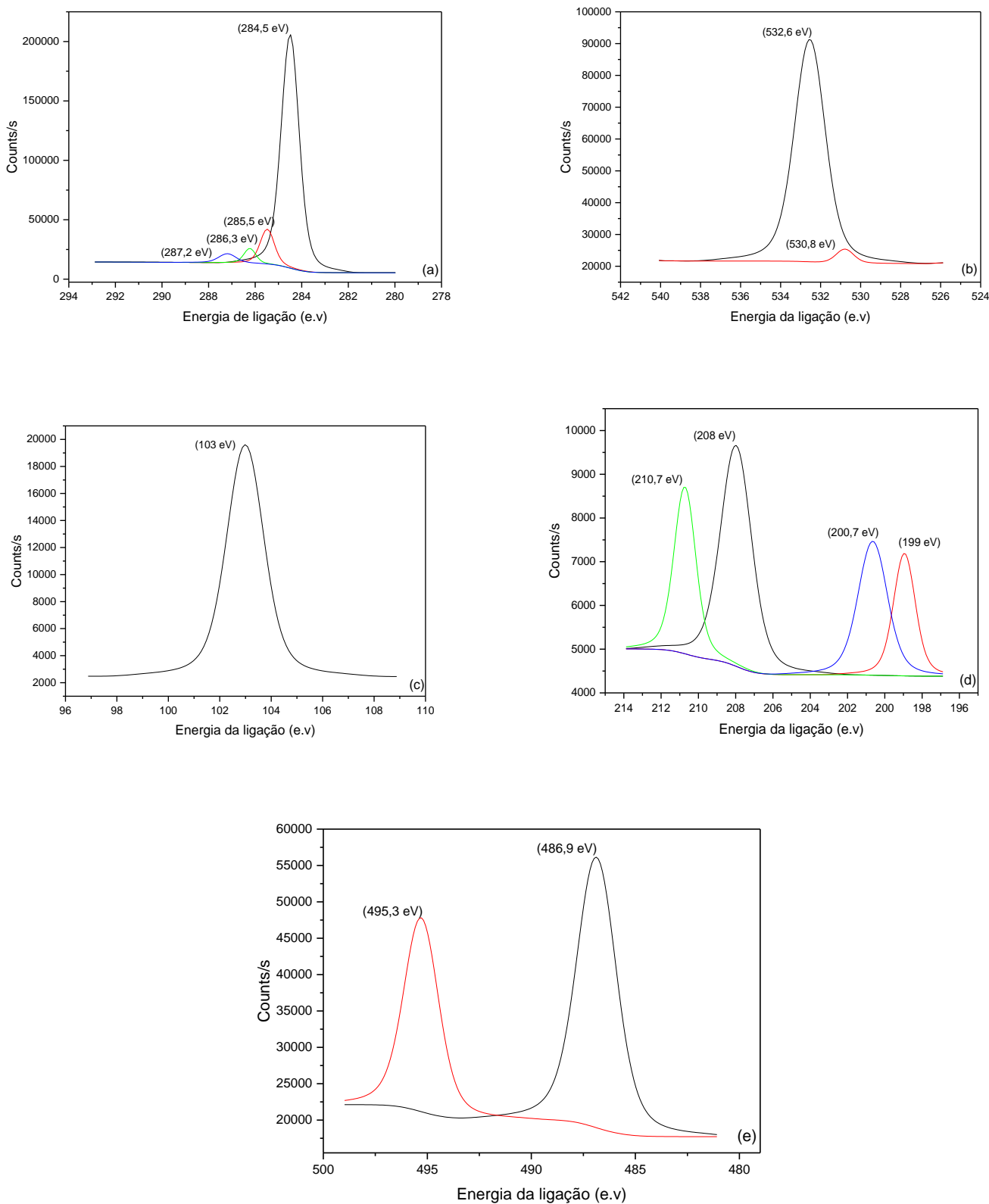

Figura 4. Espectros de XPS do $\mathrm{ECC} / \mathrm{SiO}_{2}-\mathrm{SnO}_{2}-\mathrm{Nb}_{2} \mathrm{O}_{5}$ das regiões (a) $\mathrm{C} 1 \mathrm{~s}$; (b) O 1s; (c) Si 2p; e (d) $\mathrm{Nb} 3 \mathrm{~d}$

\subsection{Difração de Raios X (DRX)}

Nas Figuras $5 a$ e $5 b$ estão representados os DRX dos sistemas em estudo. Na Figura $5 a$, pode -se observar que o $\mathrm{ECC} / \mathrm{SiO}_{2}$ apresentou apenas o pico $2 \theta$ intenso em $26,6^{\circ}$, o qual é característico de grafite cristalino (cartão no 26-1076 JCPDS Joint Committee on Powder Diffraction Standards), com índices de Miller (002). Os sistemas $\mathrm{ECC} / \mathrm{SiO}_{2}-\mathrm{SnO}_{2}, \quad \mathrm{ECC} / \mathrm{SiO}_{2}-\mathrm{Nb}_{2} \mathrm{O}_{5}$ e $\mathrm{ECC} / \mathrm{SiO}_{2}-\mathrm{SnO}_{2}-\mathrm{Nb}_{2} \mathrm{O}_{5}$ apresentaram este mesmo pico, cuja posição se manteve em função da dopagem com os óxidos, como observado nos demais difratogramas da Figura 5 . No difratograma do $\mathrm{ECC} / \mathrm{SiO}_{2}-\mathrm{SnO}_{2}$ foram identificados planos referentes a fase cassiterita do óxido de estanho, com os picos localizados em $34,0^{\circ} ; 38,1^{\circ} ; 51,9^{\circ} ; 54,8^{\circ} ; 62,0^{\circ} ; 64,9^{\circ} ; 66,1^{\circ}$, 
correspondentes ao planos (101), (111), (211), (220), (310), (112), (301), respectivamente, com base na carta cristalográfica no 72-1147 1076 ICDD. Na Figura $5 b$, pode ser observado que $\mathrm{ECC} / \mathrm{SiO}_{2}-\mathrm{Nb}_{2} \mathrm{O}_{5}$ e $\mathrm{ECC} / \mathrm{SiO}_{2}-\mathrm{SnO}_{2}-\mathrm{Nb}_{2} \mathrm{O}^{5}$ apresentam um pico em $10,8^{\circ}$ referente ao óxido de carbono $\mathrm{C}_{6} \mathrm{O}$ ficha (cartão no 47-0787 JCPDS). Para todos os difratogramas, no entanto não foi possível obter informações relevantes acerca dos óxidos adicionados com predominância das fases referentes ao grafite.
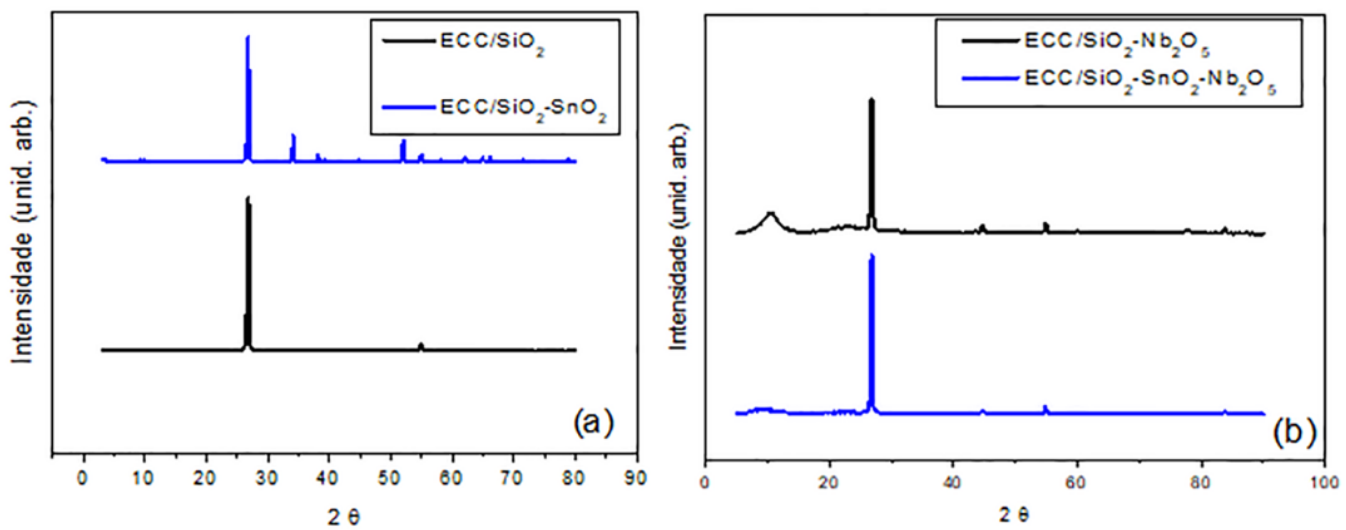

Figura 5. Padrão de difração de raios $X$ para os materiais (a) $\mathrm{ECC} / \mathrm{SiO}_{2}$ e $\mathrm{ECC} / \mathrm{SiO}_{2}-\mathrm{SnO}_{2}$ e (b) $\mathrm{ECC} / \mathrm{SiO}_{2}-\mathrm{Nb}_{2} \mathrm{O}_{5}$ e $\mathrm{ECC} / \mathrm{SiO}_{2}-\mathrm{SnO}_{2}-\mathrm{Nb}_{2} \mathrm{O}_{5}$

De forma a obter informações relevantes a respeito da estrutura cristalina dos óxidos incorporados na rede da sílica foram obtidos difratogramas de raios $X$ de sistemas sem a presença de grafite (Figura 6).

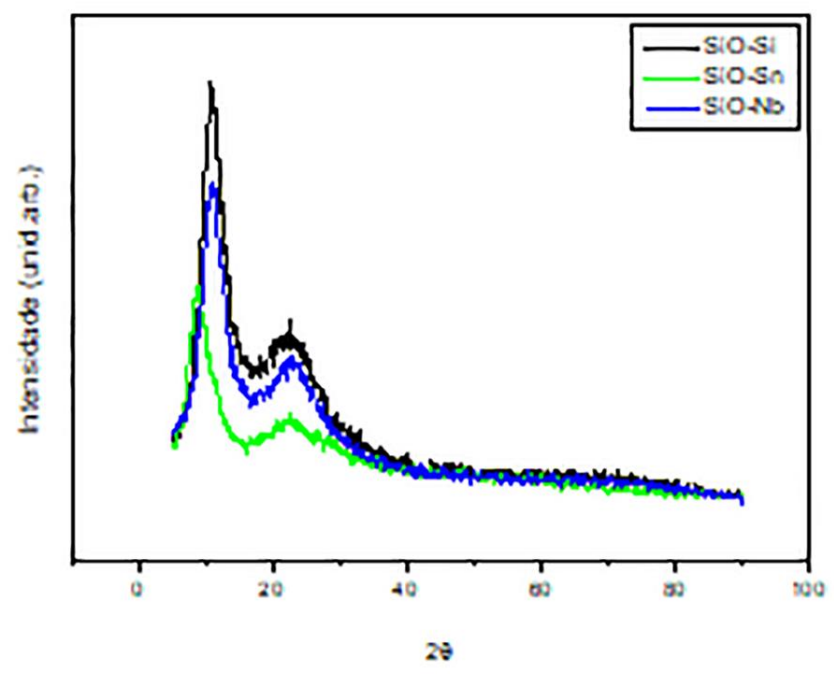

Figura 6. Padrão de difração de raios para os sistemas $\mathrm{SiO}_{2}, \mathrm{SiO}_{2}-\mathrm{SnO}_{2}, \mathrm{SiO}_{2}-\mathrm{Nb}_{2} \mathrm{O}_{5}$

O sistema $\mathrm{SiO}_{2}$ é referente a síntese proveniente do processo sol-gel sem a presença dos precursores inorgânicos $\left(\left(\mathrm{C}_{4} \mathrm{H}_{11}\right)_{2} \mathrm{Sn}\left(\mathrm{OCOCH}_{3}\right)_{2}\right.$ e $\left.\mathrm{NbCl}_{5}\right)$, sendo os demais $\mathrm{SiO}_{2}-\mathrm{SnO}_{2}$ e $\mathrm{SiO}_{2}-\mathrm{Nb}_{2} \mathrm{O}_{5}$ com a presença destes.
Em ambos os sistemas, visualizou-se apenas uma estrutura amorfa com picos alargados entre $20^{\circ}$ e $30^{\circ}$, característicos de matrizes de sílica não cristalinas. ${ }^{38}$ Independente dos precursores inorgânicos adicionados, os 
difratogramas sem a presença de grafite apresentaram estrutura amorfa o que sugere que a cristalinidade observada nos difratogramas da Figura 5 , é proveniente do grafite presente nos materiais. Alguns trabalhos da literatura descrevem a obtenção da presença de picos $r$ eferentes a cassiterita, fase cristalina do óxido de estanho a partir de $450{ }^{\circ} \mathrm{C}^{39}$ entretanto também é descrita definição dos picos em temperaturas acima de $1000{ }^{\circ} \mathrm{C}$, principalmente quando o $\mathrm{SnO}_{2}$ é incorporado em materiais de sílica. ${ }^{40,41}$. A fase tetragonal do nióbio pode ser observada em temperaturas acima de $1100{ }^{\circ} \mathrm{C}^{42} 3.5$. Caracterização Morfológica por Microscopia Eletrônica de Varredura com Fonte de Emissão de Campo (MEV/FEG)

Na Figura 7, estão apresentadas as imagens de MEV dos compósitos. A partir das imagens obtidas, pode-se observar que para todos os sistemas um material heterogêneo com variação no tamanho das partículas e a presença de lamelas referente ao carbono grafite, além da ausência de um padrão característico ou representativo.
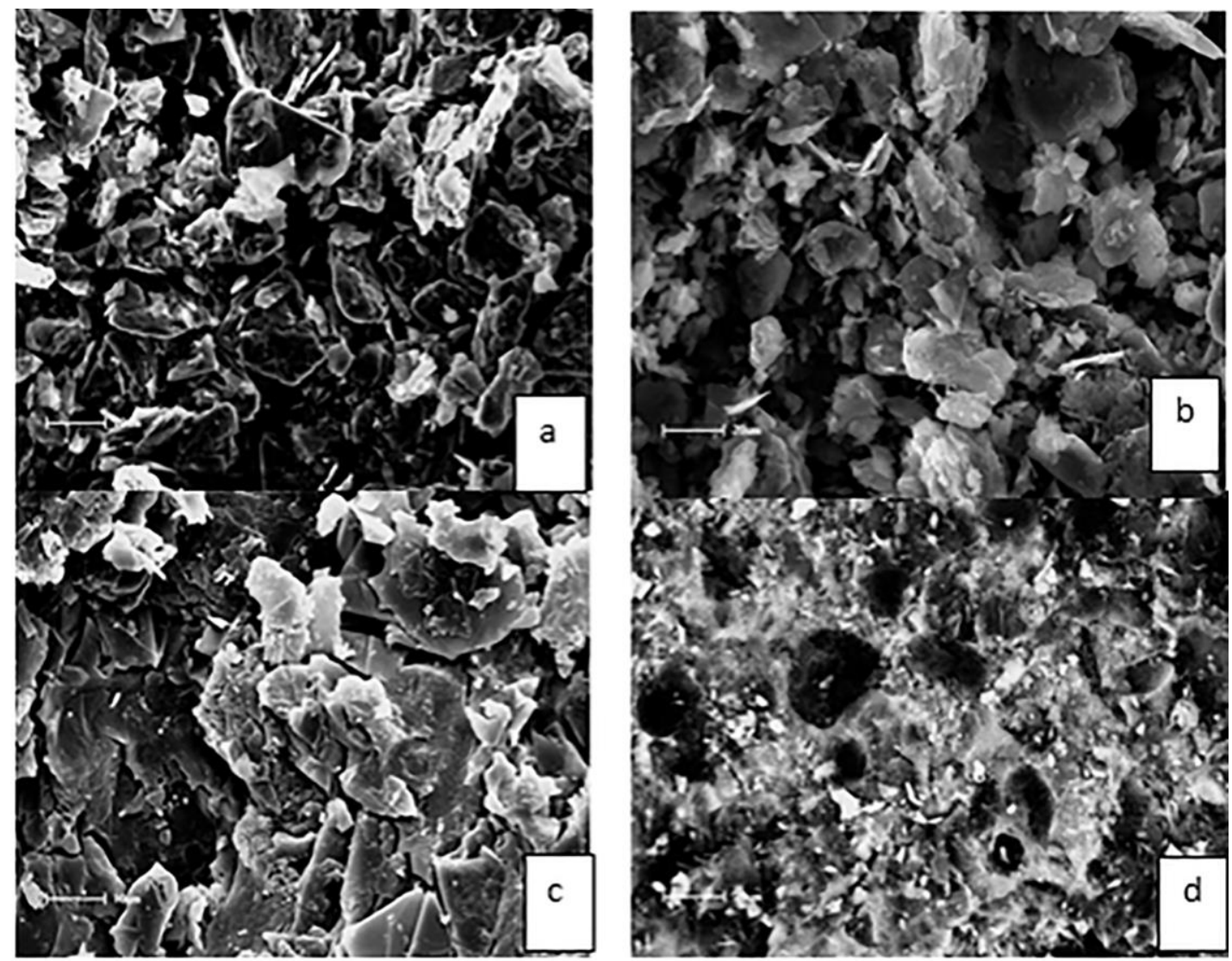

Figura 7. Análise por MEV dos compósitos $\mathrm{ECC} / \mathrm{SiO}_{2}$ (a) $\mathrm{ECC} / \mathrm{SiO}_{2}-\mathrm{SnO}_{2}$ (b) $\mathrm{ECC} / \mathrm{SiO}_{2}-\mathrm{Nb}_{2} \mathrm{O}_{5}$ (c) $\mathrm{ECC} / \mathrm{SiO}_{2}-\mathrm{SnO}_{2}-\mathrm{Nb}_{2} \mathrm{O}_{5}(\mathrm{~d})$. Aumento de $500 \mathrm{X}$

As análises de EDS para o $\mathrm{ECC} / \mathrm{SiO}_{2}-\mathrm{Nb}_{2} \mathrm{O}_{5} \mathrm{e}$ $\mathrm{ECC} / \mathrm{SiO}_{2}-\mathrm{SnO}_{2}-\mathrm{Nb}_{2} \mathrm{O}_{5}$ estão mostradas nas Figuras 8 e 9 , respectivamente, com o objetivo de verificar o grau de dispersão dos óxidos de nióbio e de estanho na rede da sílica. Pode-se observar que a incorporação dos óxidos ocorreu de forma homogênea e bem dispersa. Tal fato constitui um fator positivo, pois a dispersão uniforme dos óxidos favorece a aplicação deste material como trocador iônico e consequentemente no desenvolvimento de eletrodos de carbono cerâmico modificados com diferentes espécies eletroativas. A distribuição homogênea e efetiva dos modificadores sobre a superfície dos eletrodos possibilita a obtenção de respostas 
eletroquímicas mais reprodutíveis e estáveis. Para $0 \quad \mathrm{ECC} / \mathrm{SiO}_{2}-\mathrm{SnO}_{2}-\mathrm{Nb}_{2} \mathrm{O}_{5}$ (Figura 9) observou-se pelo mapeamento que todo o estanho e o nióbio presentes no compósito, mostraram-se bem distribuídos pela superfície. Levando-se em consideração a distribuição dos óxidos pela rede da sílica, de uma forma geral, em todos os compósitos verificou-se que o estanho e o nióbio se encontram amplamente distribuídos pela estrutura, não havendo formação de "ilhas" isoladas de $\mathrm{SnO}_{2}$ ou $\mathrm{Nb}_{2} \mathrm{O}_{5}$ no material, indicando assim, a formação das ligações Si-O-Sn ou Si-O-Nb o que corrobora com o observado a partir das análises de FTIR e XPS.
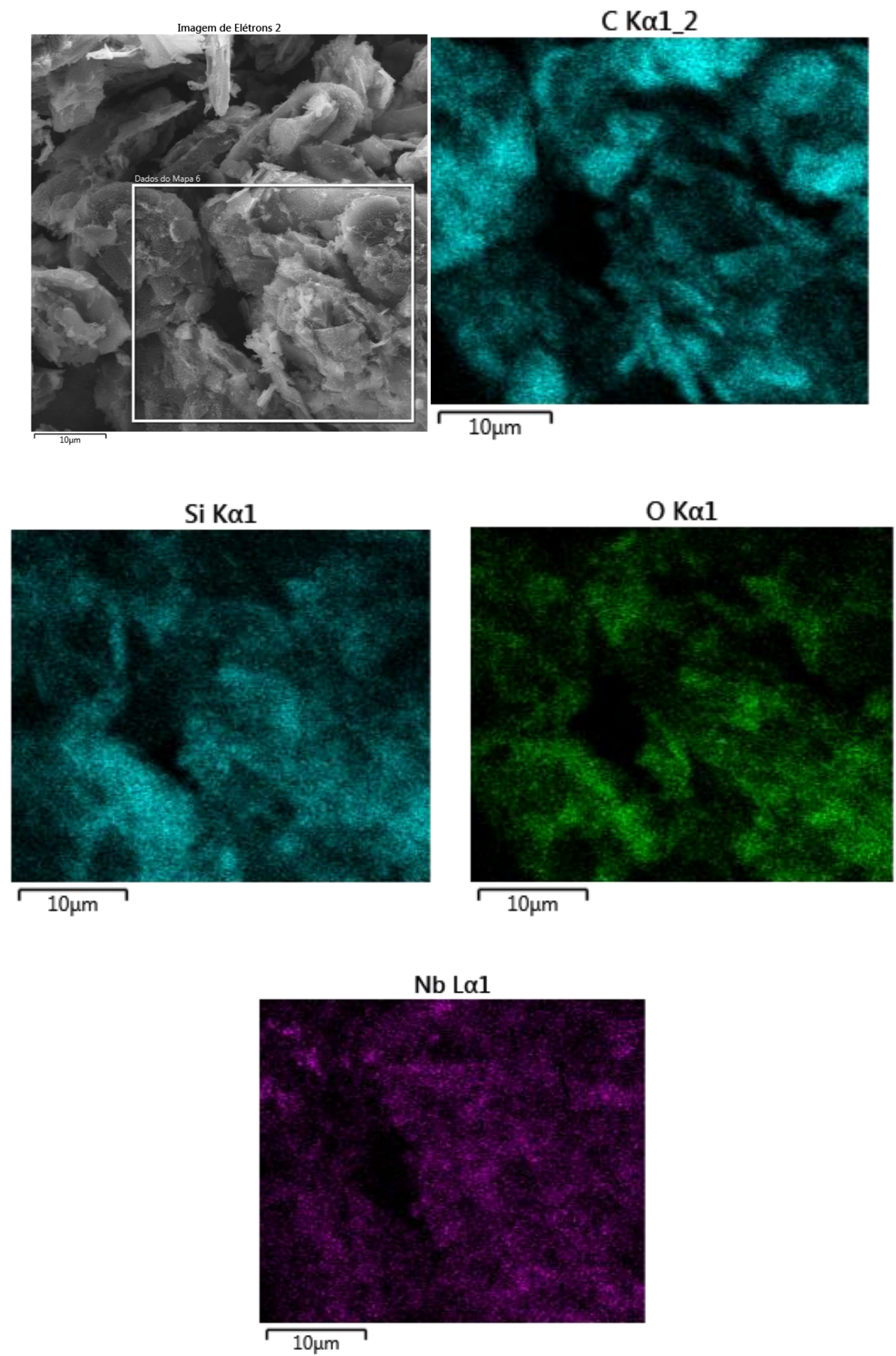

Figura 8. $\mathrm{MEV} / \mathrm{EDS}$ do $\mathrm{ECC} / \mathrm{SiO}_{2}-\mathrm{Nb}_{2} \mathrm{O}_{5}$. Mapeamento de $\mathrm{C}$; $\mathrm{Si} ; \mathrm{Nb}$; O e $\mathrm{Sn}(2 \mathrm{KX})$ 


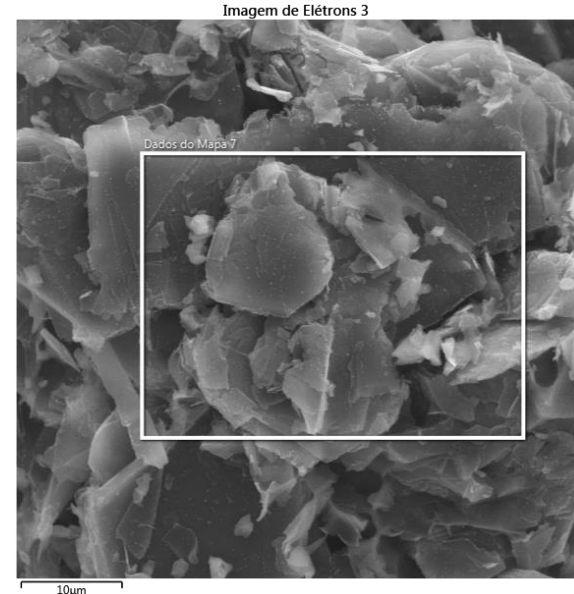

Si K $\alpha 1$

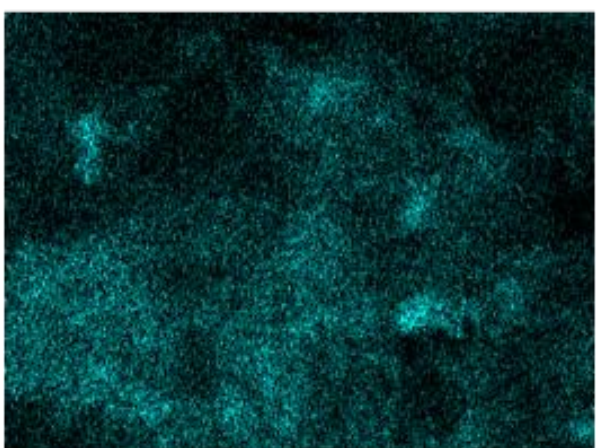

$10 \mu \mathrm{m}$

$\mathrm{O} \mathrm{K} \alpha 1$

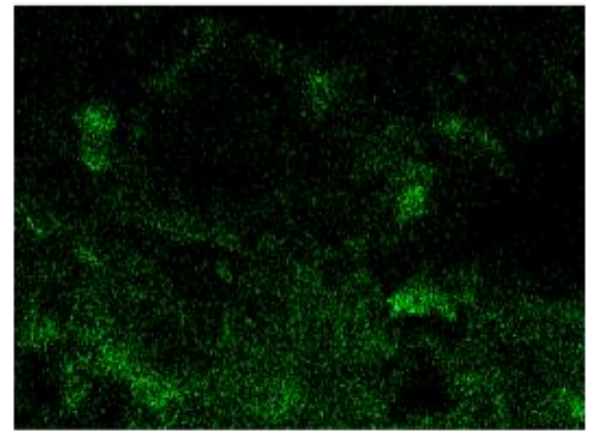

$10 \mu \mathrm{m}$

\section{$\mathrm{C} K \alpha 12$}

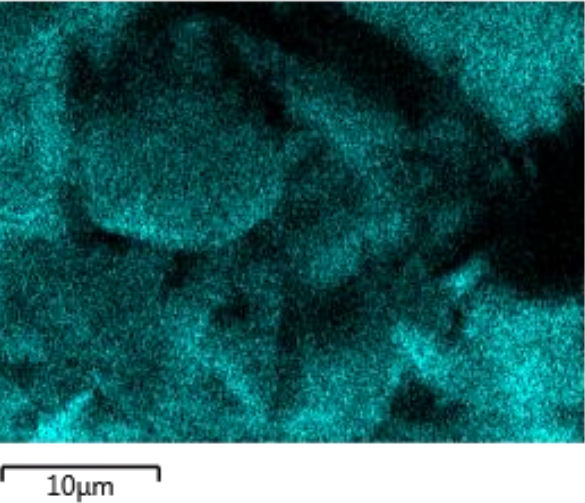

$\mathrm{Nb} \operatorname{L} \alpha 1$

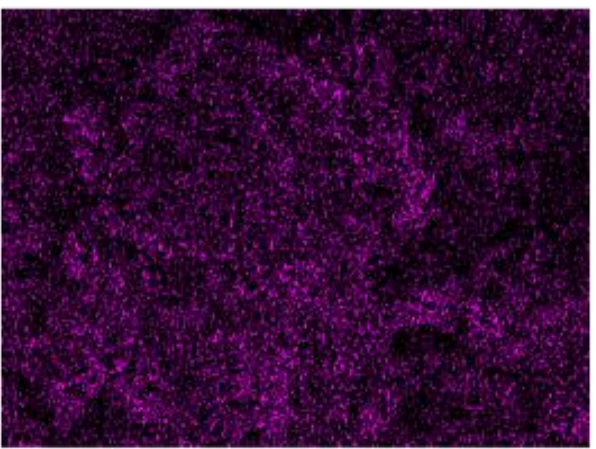

$$
10 \mu \mathrm{m}
$$

Sn $L \alpha 1$

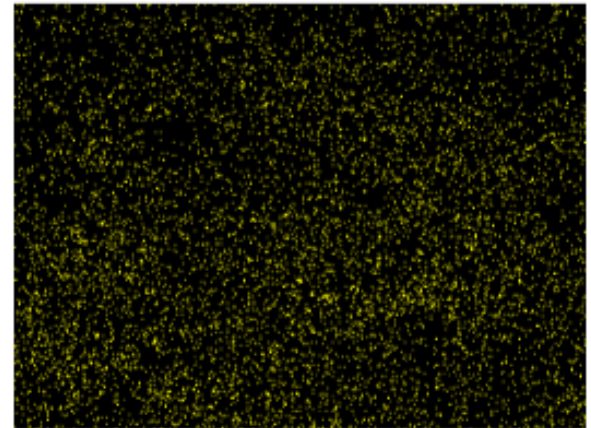

$10 \mu \mathrm{m}$

Figura 9. $\mathrm{MEV} / \mathrm{EDS}$ do $\mathrm{ECC} / \mathrm{SiO}_{2}-\mathrm{SnO}_{2}-\mathrm{Nb}_{2} \mathrm{O}_{5}$ Mapeamento $\mathrm{C}$; $\mathrm{Si}$; $\mathrm{Nb}$; O e $\mathrm{Sn}(2 \mathrm{KX}$ )

3.6. Estudos voltamétricos dos compósitos $\mathrm{ECC} / \mathrm{SiO}_{2}-\mathrm{SnO}_{2}, \mathrm{ECC} / \mathrm{SiO}_{2}-\mathrm{Nb}_{2} \mathrm{O}_{5} \mathrm{e}$ $\mathrm{ECC} / \mathrm{SiO}_{2}-\mathrm{SnO}_{2}-\mathrm{Nb}_{2} \mathrm{O}_{5}$

De modo a verificar a influência da adição de diferentes precursores inorgânicos na rede da sílica nas propriedades eletroquímicas dos ECC foram realizados primeiramente estudos de voltametria cíclica na presença da molécula sonda $\left[\mathrm{Fe}(\mathrm{CN})_{6}\right]^{3-/ 4-}$, uma vez que na ausência desta os eletrodos não apresentaram resposta eletroquímica. $\mathrm{Na}$ Figura 10, estão 
apresentados os voltamogramas obtidos para $\mathrm{ECC} / \mathrm{SiO}_{2}-\mathrm{SnO}_{2}, \mathrm{ECC} / \mathrm{SiO}_{2}-\mathrm{Nb}_{2} \mathrm{O}_{5}$ e $\mathrm{ECC} / \mathrm{SiO}_{2}-$ $\mathrm{SnO}_{2}-\mathrm{Nb}_{2} \mathrm{O}_{5}$.

Pode ser observado a partir dos voltamogramas e dos dados da Tabela 3 que o sistema $\mathrm{ECC} / \mathrm{SiO}_{2}-\mathrm{SnO}_{2}$ apresentou um valor de corrente superior ao sistema $\mathrm{ECC} / \mathrm{SiO}_{2}$ com um menor valor de $\triangle \mathrm{Ep}$. Tal comportamento pode ser atribuído ao fato do $\mathrm{SnO}_{2}$ estar homogeneamente disperso pela estrutura do ECC e consequentemente este proporciona uma diminuição significativa na resistência elétrica da matriz de sílica. ${ }^{25}$ No entanto, os sistemas $\mathrm{ECC} / \mathrm{SiO}_{2}-\mathrm{Nb}_{2} \mathrm{O}_{5}$ e $\mathrm{ECC} / \mathrm{SiO}_{2}-\mathrm{SnO}_{2}-$
$\mathrm{Nb}_{2} \mathrm{O}_{5}$ apresentaram os melhores parâmetros eletroquímicos, em particular, o $\mathrm{ECC} / \mathrm{SiO}_{2}-$ $\mathrm{SnO}_{2}-\mathrm{Nb}_{2} \mathrm{O}_{5}$ exibiu maiores valores de resposta de corrente, devido a combinação das propriedades dos óxidos de nióbio e estanho na matriz dos eletrodos de carbono cerâmico. É conhecido da literatura que a adição de óxido de nióbio em cerâmicas a base de $\mathrm{SnO}_{2}$ proporciona um aumento da condutividade elétrica das mesmas. Tal aumento na condutividade é atribuído ao fato do íon pentavalente atuar como um doador de elétrons no retículo do $\mathrm{SnO}_{2}$, uma vez que de $\mathrm{Sn}$ são criadas quando o $\mathrm{Nb}^{5+}$ substitui os íons $\mathrm{Sn}^{4+} \cdot 16$

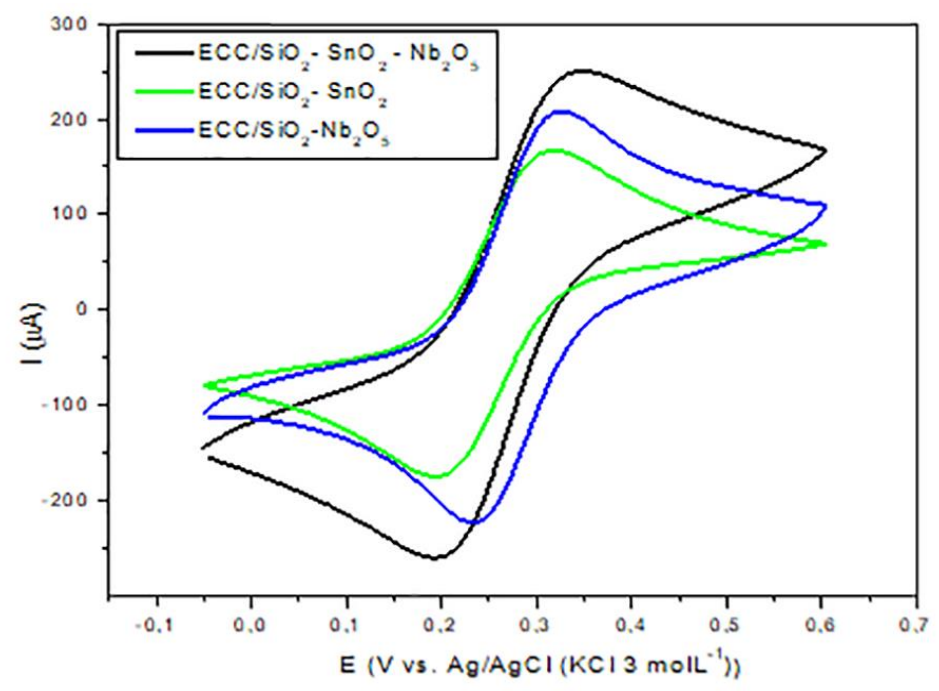

Figura 10. Voltamogramas cíclicos dos sistemas $\mathrm{ECC} / \mathrm{SiO}_{2}-\mathrm{SnO}_{2}, \mathrm{ECC} / \mathrm{SiO}_{2}-\mathrm{Nb}_{2} \mathrm{O}_{5}$ e ECC/SiO${ }_{2}-$ $\mathrm{SnO}_{2}-\mathrm{Nb}_{2} \mathrm{O}_{5}$, realizados na presença de $\left[\mathrm{Fe}(\mathrm{CN})_{6}\right]^{3-/ 4-} 5,0 \mathrm{mmol}, v=50 \mathrm{mV} \cdot \mathrm{s}^{-1}$

Na Tabela 3, está uma comparação dos parâmetros voltamétricos de todos os sistemas estudados.

Pode ser observado a partir dos voltamogramas e dos dados da Tabela 3 que o sistema $\mathrm{ECC} / \mathrm{SiO}_{2}-\mathrm{SnO}_{2}$ apresentou um valor de corrente superior ao sistema $\mathrm{ECC} / \mathrm{SiO}_{2} \mathrm{com}$ um menor valor de $\triangle \mathrm{Ep}$. Tal comportamento pode ser atribuído ao fato do $\mathrm{SnO}_{2}$ estar homogeneamente disperso pela estrutura do ECC e consequentemente este proporciona uma diminuição significativa na resistência elétrica da matriz de sílica. ${ }^{25}$ No entanto, os sistemas $\mathrm{ECC} / \mathrm{SiO}_{2}-\mathrm{Nb}_{2} \mathrm{O}_{5}$ e $\mathrm{ECC} / \mathrm{SiO}_{2}-\mathrm{SnO}_{2}-$ $\mathrm{Nb}_{2} \mathrm{O}_{5}$ apresentaram os melhores parâmetros eletroquímicos, em particular, o $\mathrm{ECC} / \mathrm{SiO}_{2-}$
$\mathrm{SnO}_{2}-\mathrm{Nb}_{2} \mathrm{O}_{5}$ exibiu maiores valores de resposta de corrente, devido a combinação das propriedades dos óxidos de nióbio e estanho na matriz dos eletrodos de carbono cerâmico. É conhecido da literatura que a adição de óxido de nióbio em cerâmicas a base de $\mathrm{SnO}_{2}$ proporciona um aumento da condutividade elétrica das mesmas. Tal aumento na condutividade é atribuído ao fato do íon pentavalente atuar como um doador de elétrons no retículo do $\mathrm{SnO}_{2}$, uma vez que de Sn são criadas quando o $\mathrm{Nb}^{5+}$ substitui os íons $\mathrm{Sn}^{4+} \cdot 16$

Estudos em diferentes velocidades de varredura realizados para definir qual $o$ processo de transferência de massa ocorre na 
interface eletrodo-solução, evidenciaram que para todos os sistemas $\left(\mathrm{ECC} / \mathrm{SiO}_{2}-\mathrm{SnO}_{2}\right.$, $\mathrm{ECC} / \mathrm{SiO}_{2}-\mathrm{Nb}_{2} \mathrm{O}_{5}$ e $\left.\mathrm{ECC} / \mathrm{SiO}_{2}-\mathrm{SnO}_{2}-\mathrm{Nb}_{2} \mathrm{O}_{5}\right)$ uma correlação linear de Ipa em função da raiz quadrada da velocidade foi obtida, indicando que o processo de transferência de elétrons (Figuras S1, S2 e S3, respectivamente) é controlado pela difusão das espécies na interface do eletrodo. Adicionalmente, todos os sistemas constituíram um processo quasereversível, onde o $\Delta \mathrm{Ep}$ aumentou com o aumento da velocidadFoi verificada a repetibilidade da resposta voltamétrica dos ECC modificados com os óxidos em função do número de ciclos voltamétricos na presença da molécula sonda, onde verificou-se que após a varredura de 100 ciclos ocorreu uma variação do Ipa de $1,45 \%$ e Ipc de 0,044 \% para o $\mathrm{ECC} / \mathrm{SiO}_{2}-\mathrm{SnO}_{2}$, Ipa $=4,73 \%$ e do Ipc= $3,86 \%$ para $\mathrm{ECC} / \mathrm{SiO}_{2}-\mathrm{Nb}_{2} \mathrm{O}_{5}$, Ipa $=2,72 \%$ e Ipc $=3,61 \%$ para $\mathrm{ECC} / \mathrm{SiO}_{2}-\mathrm{SnO}_{2}-\mathrm{Nb}_{2} \mathrm{O}_{5}$, indicando a alta estabilidade de respostas dos ECCs. Tais resultados demonstram a potencialidade de utilização destes materiais no desenvolvimento de sensores.

Tabela 3. Parâmetros voltamétricos obtidos para os eletrodos compósitos obtidos

\begin{tabular}{lcccc}
\hline Eletrodo & Ipa/ $\boldsymbol{\mu A}$ & $\mathbf{I p c} / \boldsymbol{\mu A}$ & ]Ep (mV) & Ipa/Ipc (mv) \\
\hline $\mathrm{ECC} / \mathrm{SiO}_{2}$ & 147 & 145,9 & 132 & 1,00 \\
$\mathrm{ECC} / \mathrm{SiO}_{2}-\mathrm{SnO}_{2}$ & 164,7 & 160,0 & 123 & 1,021 \\
$\mathrm{ECC} / \mathrm{SiO}_{2}-\mathrm{Nb}_{2} \mathrm{O}_{5}$ & 196 & 190 & 94 & 1,022 \\
$\mathrm{ECC} / \mathrm{SiO}_{2}-\mathrm{SnO}_{2}-\mathrm{Nb}_{2} \mathrm{O}_{5}$ & $\mathbf{2 1 4 , 4}$ & $\mathbf{2 2 4 , 7 0}$ & 150 & 0,96 \\
\hline
\end{tabular}

\section{Conclusões}

Os espectros de FTIR indicaram a incorporação dos óxidos de $\mathrm{Nb}$ e Sn na rede de sílica uma vez que os espectros dos sistemas $\mathrm{ECC} / \mathrm{SiO}_{2}-\mathrm{SnO}_{2}$ e $\mathrm{ECC} / \mathrm{SiO}_{2}-\mathrm{SnO}_{2}-\mathrm{Nb}_{2} \mathrm{O}_{5}$ apresentaram bandas próximas a $620 \mathrm{~cm}^{-1}$ referentes a ligação $\mathrm{M}-\mathrm{O}-\mathrm{Si}$ (onde $\mathrm{M}=\mathrm{Nb}$ ou $\mathrm{Sn})$. A partir da técnica de espectroscopia de Raman, pode-se constatar que a medida em que se adicionou-se precursores inorgânicos na rede da sílica, aumentou-se o grau de desordem em comparação ao sistema $\mathrm{ECC} / \mathrm{SiO}_{2}$, sem a presença destes.

As análises de microscopia eletrônica de varredura (MEV/EDS) dos sistemas obtidos, mostraram que a incorporação dos óxidos de nióbio e óxido de estanho ocorreu de forma homogênea e dispersa pela superfície da matriz.

Os eletrodos de carbono cerâmico, onde houve adição de precursores inorgânicos apresentaram melhores respostas voltamétricas que os sistemas sem a adição destes $\left(\mathrm{ECC} / \mathrm{SiO}_{2}\right)$. Tais resultados sugerem que a adição dos óxidos proporcionou menor resistência elétrica da matriz do compósito. Todos os sistemas constituíram um processo quase-reversível, característico de um processo difusional de transferência de massa, e ambas se apresentaram estáveis após a ciclagem de 100 ciclos, com variação de Ipa e Ipc abaixo de $5 \%$. Tais resultados demonstram a potencialidade de utilização destes materiais no desenvolvimento de sensores.

\section{Referências Bibliográficas}

${ }^{1}$ Gun, G.; Tsionsky, M; Lev, O. Voltammetric studies of composite ceramic carbon working electrodes. Analytica Chimica Acta 1994, 294, 261. [CrossRef]

${ }^{2}$ Alfaya, A. A. S.; Kubota, L. T. A utilização de materiais obtidos pelo processo de sol-gel na construção de biossensores. Química Nova 2002, 25, 835. [CrossRef]

${ }^{3}$ Kutner, W.; Wang, J.; L'her, M.; Buck, R. P. 
Analytical aspects of chemically modified electrodes: classification, critical evaluation and recommendations. Pure Applied Chemistry 1998, 70, 1301. [CrossRef]

${ }^{4}$ Machado, S.; Calaça, G. N.; da Silva, J. P.; de Araujo, M. P.; Boeré, R. T.; Pessôa, C. A.; Wohnrath, K. Electrochemical Characterization of a Carbon Ceramic Electrode Modified with a Ru(II) Arene Complex and Its Application as Voltammetric Sensor for Paracetamol. Journal of The Electrochemical Society 2017, 164, B314. [CrossRef]

${ }^{5}$ Ciórcero, J. R.; Calaça, G. N.; Pessôa, C. A. Carbon ceramic electrodes modified with mixed oxides $\mathrm{SiO} 2 / \mathrm{SnO} 2$ for determination of levofloxacin. Journal of Solid State Electrochemistry 2017, 22, 1403. [CrossRef]

${ }^{6}$ Heidari, H.; Habibi, E. Lead-doped carbon ceramic electrode as a renewable surface composite electrode for the preparation of lead dioxide film and detection of I-tyrosine. Journal of the Iranian Chemical Society 2018, 15, 885. [CrossRef]

${ }^{7}$ Yu, H.; Jian, X.; Jin, J.; Zheng, X.; Liu, R.; Qi, G. Nonenzymatic sensing of glucose using a carbon ceramic electrode modified with a composite film made from copper oxide, overoxidized polypyrrole and multi-walled carbon nanotubes. Microchimica Acta 2014, 182, 157. [CrossRef]

${ }^{8}$ Sheng, Q.; Yu, H.; Zheng, J. Hydrogen peroxide determination by carbon ceramic electrodes modified with pyrocatechol violet. Electrochimica Acta 2007, 52, 7300. [CrossRef]

${ }^{9}$ Zhu, L.; Tian, C.; Zhai, J.; Yang, R. Sol-gel derived carbon nanotubes ceramic composite electrodes for electrochemical sensing. Sensors and Actuators B: Chemical 2007, 125, 254. [CrossRef]

${ }^{10}$ Caldas, E. M.; Novatzky, D.; Deon, M.; de Menezes, E. W.; Hertz, P. F.; Costa, T. M. H.; Benvenutti, E. V. Pore size effect in the amount of immobilized enzyme for manufacturing carbon ceramic biosensor. Microporous and Mesoporous Materials 2017, ${ }^{19}$ Francisco, M. S. P.; Cardoso, W. S.; Kubota, L. T.; Gushikem, Y. Electrocatalytic oxidation of

\section{7, 95.[CrossRef]}

${ }^{11}$ Sampath, S.; Pankratov, Irena; Gun, J.; Lev, O. Sol-gel derived ceramic-carbon enzyme electrodes: glucose oxidase as a test case, Journal of Sol-Gel Science and Technology 1996, 7, 123.[CrossRef]

${ }^{12}$ Lei, C. X.; Huc, S. Q.; Gaoa, N; Shena, G.L; Yua, R. Q. An amperometric hydrogen peroxide biosensor based on immobilizing horseradish peroxidase to a nano-Au monolayer supported by sol-gel derived carbon ceramic electrode. Bioelectrochemistry 2004, 65, 33. [CrossRef]

${ }^{13}$ Rohani, T.; Ali Taher, M. Preparation of a carbon ceramic electrode modified by 4-(2pyridylazo)-resorcinol for determination of trace amounts of silver. Talanta 2010, 80 1827. [CrossRef]

${ }^{14}$ Razmi, H.; Habibi, Es.; Heidari, H. Electrocatalytic oxidation of methanol and ethanol at carbon ceramic electrode modified with platinum nanoparticles. Electrochimica Acta 2008, 53, 8178. [CrossRef]

${ }^{15}$ Gao, Y.; Wang, L.; Zhang, Y.; Zou, L.; Li, G.; Ye, B. Highly sensitive determination of gallic acid based on a Pt nanoparticle decorated polyelectrolyte-functionalized graphene modified electrode. Analytical Methods 2016, 8, 8474. [CrossRef]

${ }^{16}$ Leite, D. R., Mazali, I. O., Aguiar, E. C., Las, W. C., Cilense, $\mathrm{M}$. The effect of $\mathrm{Sb}$ and $\mathrm{Nb}$ on the electrical conductivity of tin dioxide based ceramics. Journal of Materials Science 2006, 41, 6256. [CrossRef]

${ }^{17}$ Arguello, J.; Magosso, H. A.; Landers, R.; Pimentel, V. L.; Gushikem, Y. Synthesis, characterization and electroanalytical application of a new $\mathrm{SiO}_{2} / \mathrm{SnO}_{2}$ carbon ceramic electrode. Electrochimica Acta 2010, 56, 340. [CrossRef]

${ }^{18}$ Zaitseva, G.; Gushikem, Y.; Ribeiro, E.S.; Rosatto, S.S. Electrochemical property of methylene blue redox dye immobilized on porous silica-zirconia-antimonia mixed oxide. Electrochimica Acta 2002, 47, 1469. [CrossRef]

phenolic compounds using an electrode modified with $\mathrm{Ni}(\mathrm{II})$ porphyrin adsorbed on $\mathrm{SiO}_{2} / \mathrm{Nb}_{2} \mathrm{O}_{5}-$ 
phosphate synthesized by the sol-gel method. Journal of Electroanalytical Chemistry 2007, 609, 61. [CrossRef]

${ }^{20}$ Pereira, A.C.; Santos, A.S.; Kubota, L.T. OPhenylenediamine adsorbed onto silica gel modified with niobium oxide for electrocatalytic NADH oxidation. Electrochimica Acta 2003, 48, 3541. [CrossRef]

${ }^{21}$ Maroneze, C. M.; Arenas, L. T.; Luz, R. C. S.; Benvenutti, E. V.; Landers, R.; Gushikem, Y. Meldola blue immobilized on a new $\mathrm{SiO}_{2} /$ $\mathrm{TiO}_{2}$ /graphite composite for electrocatalytic oxidation of NADH. Electrochimica Acta 2008, 53, 4167. [CrossRef]

22 Mora-Gómez, J.; García-Gabaldón, M.; Ortega, E.; Sánchez-Rivera, M-J.; Mestre, S.; Pérez-Herranz, V. Evaluation of new ceramic electrodes based on $\mathrm{Sb}$-doped $\mathrm{SnO}_{2}$ for the removal of emerging compounds present in wastewater. Ceramics International 2018, 44, 2216. [CrossRef]

${ }^{23}$ Grieken, R. V.; Martos, C.; Sánchez, M.; Serrano, D. P.; Melero, J. A.; Iglesias, J.; Cubero, A. G. Synthesis of Sn-silicalite from hydrothermal conversion of $\mathrm{SiO}_{2}-\mathrm{SnO}_{2}$ xerogels Microporous and Mesoporous Materials 2009, 119, 176. [CrossRef]

${ }^{24}$ Arguello, J.; Magosso, H. A.; Landers, R.; Pimentel, V. L.; Gushikem, Y. Synthesis, characterization and electroanalytical application of a new $\mathrm{SiO}_{2} / \mathrm{SnO}_{2}$ carbon ceramic electrode. Electrochimica Acta 2010, 56, 340. [CrossRef]

${ }^{25}$ Canevari, T.C.; Arguello, J.; Francisco, M.S.P.; Gushikem, Y. Cobalt phthalocyanine prepared in situ on a sol-gel derived $\mathrm{SiO}_{2} / \mathrm{SnO}_{2}$ mixed oxide. Journal of Electroanalytical Chemistry 2007, 609, 61. [CrossRef]

26 Lopes, O. F.; Mendonça, V. R.; Silva, F. B. F.; Paris, E. C.; Ribeiro, C. Óxidos de Nióbio: uma visão sobre a síntese do $\mathrm{Nb}_{2} \mathrm{O}_{5}$ e sua aplicação em fotocatálise heterogênea. Química Nova 2015, 38, 106. [CrossRef]

27 Tarley, C. R. T.; de Ávila, T. C.; Segatelli, M. G.; Lima, G. F.; Peregrino, G. S.; Scheeren, C. W.; Dias, S. L. P. ; Ribeiro, E. S. J. Silica-aluminaniobia $\left(\mathrm{SiO}_{2} / \mathrm{Al}_{2} \mathrm{O}_{3} / \mathrm{Nb}_{2} \mathrm{O}_{5}\right)$ matrix obtained by the sol-gel processing method: new material for online extraction of zinc ions. Journal of the Brazilian Chemical Society 2010, 21, 1106. [CrossRef]

${ }^{28}$ Wu, Y. C.; Hamd, W.; Thune, E.; Boulle, A.; Rochas, C.; Guinebretière, R. Synthesis of tin oxide nanosized crystals embedded in silica matrix through sol-gel process using alkoxide precursors. Journal of Non-Crystalline Solids 2009, 355, 951. [CrossRef]

${ }^{29}$ Arenas, L. T.; Villis, P. C. M.; Arguello, J.; Landers, R.; Benvenutti, E. V.; Gushikem, Y. Niobium oxide dispersed on a carbon-ceramic matrix, $\mathrm{SiO}_{2} / \mathrm{C} / \mathrm{Nb}_{2} \mathrm{O}_{5}$, used as an electrochemical ascorbic acid sensor. Talanta 2010, 83, 241. [CrossRef]

${ }^{30}$ Huang, H.; Kelder, E.M.; Chen, L. Preparation and structure of silicon doped tin oxide composites using an advanced ultrasonic spray. Solid State Ionics 1998, 120, 205. [CrossRef]

${ }^{31}$ Lobo, A. O.; Martin, A.A.; Antunes, E.F.; Trava-Airoldi, V.J.; Corat, E.J. Caracterização de materiais carbonosos por espectroscopia Raman. Revista Brasileira de Aplicações de Vácuo 2005, 24, 98. [CrossRef]

32 Bolzon, L. B.; Dissertação de Mestrado, Universidade de Brasília, Brasil, 2007. [Link]

33 Oliveira, M. A; Dissertação de Mestrado, Universidade de São Paulo, Brasil, 2013. [Link]

${ }^{34}$ Pereira, E. B.; Pereira, M. M.; Lam, Y. L.; Perez, C. A. C.; Schmal, M. Synthesis and characterization of niobium oxide layers on silica and the interaction with nickel, Applied Catalysis A: General 2000, 197, 99. [CrossRef]

${ }^{35}$ Wojcieszak, R.; Jasik, A.; Monteverdi, S.; Ziolek, M.; Bettahar, M. M. Nickel niobia interaction in non classical $\mathrm{Ni} / \mathrm{Nb}_{2} \mathrm{O}_{5}$ catalysts. Journal of Molecular Catalysis A: Chemical 2006, 256, 225. [CrossRef]

${ }^{36}$ Daccà, A.; Gemme, G.; Mattera, L.; Parodi, R. XPS analysis of the surface composition of niobium for superconducting RF cavities. Applied Surface Science 1998, 126, 219. [CrossRef]

37 Gao, X.; Wachs, I. E. Titania-silica as catalysts: molecular structural characteristics and physico- 
chemical properties. Catalysis Today 1999, 51, 233. [CrossRef]

${ }^{38}$ Silveira, G.; Morais, A.; Villis, P.C.M.; Maroneze, C.M.; Gushikem, Y.; Lucho, A.M.S.; Pissetti, F.L. Electrooxidation of nitrite on a silica-cerium mixed oxide carbon paste electrode, Journal of Colloid and Interface Science 2012, 369, 302. [CrossRef]

${ }^{39}$ Ribeiro, J.; Alves, P. D. P.; Andrade, A. R. Effect of the preparation methodology on some physical and electrochemical properties of Ti/Ir $\mathrm{X}$ $\mathrm{Sn}(1-\mathrm{x}) \mathrm{O}_{2}$ materials, Journal of Material Science 2007, 42, 9293. [CrossRef]

${ }^{40}$ Kurihara, L. A.; Fujiwara, S. T.; Alfaya, R. V. S.; Gushikem, Y.; Alfaya, A. A. S.; Castro, S.C. Copper (II) adsorbed on $\mathrm{SiO}_{2} / \mathrm{SnO}_{2}$ obtained by the sol-gel processing method: application as electrochemical sensor for ascorbic acid, Journal of Colloid and Interface Science 2004, 274, 579. [CrossRef]

${ }^{41}$ Cardoso, W. S.; Francisco, M. S. P.; Lucho, A. M. S.; Gushikem, Y. Synthesis and acid properties of the $\mathrm{SiO}_{2} / \mathrm{SnO}_{2}$ mixed oxides obtained by the sol-gel process. Evaluation of immobilized copper hexacyanoferrate as an electrochemical probe. Solid State lonics 2004,167, 165. [CrossRef]

${ }^{42}$ Lin, X.; Wu, M.; Wang, Y.; Hagfeldt, A.; Ma, T. Novel counter electrode catalysts of niobium oxides supersede Pt for dye-sensitized solar cells. Chemical Communications 2011, 47, 11489. [CrossRef] 